\title{
Scaling of Movement Is Related to Pallidal $\gamma$ Oscillations in Patients with Dystonia
}

\author{
Christof Brücke, ${ }^{1}$ Julius Huebl, ${ }^{1}$ Thomas Schönecker, ${ }^{1}$ Wolf-Julian Neumann, ${ }^{1}$ Kielan Yarrow, ${ }^{4}$ Andreas Kupsch, ${ }^{1}$ \\ Christian Blahak, ${ }^{5}$ Goetz Lütjens, ${ }^{3}$ Peter Brown, ${ }^{6}$ Joachim K. Krauss, ${ }^{3}$ Gerd-Helge Schneider, ${ }^{2}$ and Andrea A. Kühn ${ }^{1}$ \\ Departments of ${ }^{1}$ Neurology and ${ }^{2}$ Neurosurgery, Campus Virchow, Charité-University Medicine Berlin, 13353 Berlin, Germany, ${ }^{3}$ Department of \\ Neurosurgery, Medizinische Hochschule, 30625 Hannover, Germany, ${ }^{4}$ Department of Psychology, City University, London EC1V 0HB, United \\ Kingdom, ${ }^{5}$ Department of Neurology, Universitätsmedizin Mannheim, University of Heidelberg, 68167 Mannheim, Germany, and ${ }^{6}$ Department of \\ Clinical Neurology, University Oxford, Oxford OX3 9DU, United Kingdom
}

Neuronal synchronization in the gamma $(\gamma)$ band is considered important for information processing through functional integration of neuronal assemblies across different brain areas. Movement-related $\gamma$ synchronization occurs in the human basal ganglia where it is centered at $\sim 70 \mathrm{~Hz}$ and more pronounced contralateral to the moved hand. However, its functional significance in motor performance is not yet well understood. Here, we assessed whether event-related $\gamma$ synchronization (ERS) recorded from the globus pallidus internus in patients undergoing deep brain stimulation for medically intractable primary focal and segmental dystonia might code specific motor parameters. Pallidal local field potentials were recorded in 22 patients during performance of a choice-reaction-time task. Movement amplitude of the forearm pronation-supination movements was parametrically modulated with an angular degree of $30^{\circ}, 60^{\circ}$, and $90^{\circ}$. Only patients with limbs not affected by dystonia were tested. A broad contralateral $\gamma$ band $(35-105 \mathrm{~Hz})$ ERS occurred at movement onset with a maximum reached at peak velocity of the movement. The pallidal oscillatory $\gamma$ activity correlated with movement parameters: the larger and faster the movement, the stronger was the synchronization in the $\gamma$ band. In contrast, the event-related decrease in beta band activity was similar for all movements. Gamma band activity did not change with movement direction and did not occur during passive movements. The stepwise increase of $\gamma$ activity with movement size and velocity suggests a role of neuronal synchronization in this frequency range in basal ganglia control of the scaling of ongoing movements.

\section{Introduction}

Neuronal synchronization in the gamma $(\gamma)$ frequency range $(>$ $30 \mathrm{~Hz}$ ) has been found in different brain areas and has been related to specific functions, such as vision, somatosensory processing, memory, and motor preparation (Bauer et al., 2006; Engel et al., 2001; Jensen et al., 2007; Schoffelen et al., 2005; Buzsáki, 2006). Synchronization of $\gamma$ band activity is considered as an efficient temporal code for implementing complex forms of information processing afforded by neuronal coherence (Varela et al., 2001). Movement-related induced $\gamma$ band activity can be recorded in the electrocorticogram from the motor cortex of patients with epilepsy contralateral to the side of the movement (Crone et al., 1998; Pfurtscheller et al., 2003) and has been localized to the contralateral human motor cortex in healthy subjects

Received July 28, 2011; revised Nov. 15, 2011; accepted Nov. 22, 2011.

Author contributions: C.Brücke, K.Y., P.B., and A.A.K. designed research; C.Brücke, J.H., and W.-J.N. performed research; C.Brücke, T.S., A.K., C.Blahak, G.L., J.K.K., G.-H.S., and A.A.K. contributed unpublished reagents/analytic tools; C.Brücke and A.A.K. analyzed data; C.Brücke, P.B., and A.A.K. wrote the paper.

A.A.K. was supported by the Else Kröner Memorial fellowship (EKMS 08/22) and C.Brücke by a PhD-scholarship of the Charité-University Medicine Berlin. A.A.K., A.K., and G.-H.S. are supported by the Deutsche Forschungsgemeinschaft (KF0 247).

Disclosure: J.K.K. is a consultant to Medtronic.

Correspondence should be addressed to Andrea A. Kühn, Department of Neurology, Campus Virchow, CharitéUniversity Medicine Berlin, Augustenburger Platz 1, 13353 Berlin, Germany. E-mail: andrea.kuehn@charite.de.

DOI:10.1523/JNEUROSCI.3860-11.2012

Copyright $\odot 2012$ the authors $\quad 0270-6474 / 12 / 321008-12 \$ 15.00 / 0$ using noninvasive magnetencephalography (MEG) (Cheyne et al., 2008). While the functional role of $\gamma$ synchronization in cortical areas is increasingly appreciated, $\gamma$ band activity in the human basal ganglia (BG) has only recently been found in patients undergoing deep brain stimulation (DBS) for severe movement disorders.

In patients with Parkinson's disease (PD), increased 60-80 $\mathrm{Hz}$ activity has been considered prokinetic (Brown, 2003) since it occurs in the subthalamic nucleus (STN) and globus pallidus internus (GPi) at rest after intake of dopaminergic medication in parallel with improvement of motor symptoms (Brown et al., 2001; Cassidy et al., 2002; Silberstein et al., 2003) and was associated with dyskinesia (Fogelson et al., 2005). Movement-related $\gamma$ synchronization has been found predominantly contralateral to the moved side in various basal ganglia nuclei and the thalamus in patients with different pathologies (Kempf et al., 2007; Androulidakis et al., 2007; Alegre et al., 2005; Brücke et al., 2008b; Liu et al., 2008; Lalo et al., 2008; Kempf et al., 2009), suggesting its general role in information processing in the BG.

However, there has been little work addressing how induced $\gamma$ band activity in the human BG relates to specific parameters of motor processing during preparation or performance of a movement. The precise function of the basal ganglia in motor control is still debated (for review, see Turner and Desmurget, 2010). One influential theory hypothesizes that BG play an important role in scaling of movements, and local inactivation of the GPi reduces 
movement amplitude, speed, and acceleration in monkeys (Horak and Anderson, 1984; Desmurget and Turner, 2008; Mink and Thach, 1991c). Similarly, pallidal DBS may induce slowness of movement in patients with dystonia (Ostrem et al., 2007; Berman et al., 2009; Blahak et al., 2011).

The aim of the present study was to explore different movement parameters such as amplitude, speed, and movement direction in relation to $\gamma$ band synchronization in the human BG. Since we set out to examine movement parameters in a state "as normal as possible," local field potential activity was recorded from the GPi in patients undergoing DBS for cervical or segmental dystonia with preserved normal hand motor function. We found a movement-related increase in pallidal synchronized $\gamma$ band activity that was related to movement amplitude and velocity, regardless of the direction of movement.

\section{Materials and Methods}

Patients and surgery. Local field potentials (LFP) from the GPi were recorded from 22 patients suffering from primary dystonia during performance of a choice-reaction-time task. Clinical details are given in Table 1. Nineteen patients were diagnosed with a focal cervical dystonia, three patients had a segmental dystonia. Eleven patients were male and 11 female. Patients took part with informed consent, the permission of the local ethics committee of the Charité, University Medicine Berlin and the Hannover Medical School and in accordance with the standards set by the Declaration of Helsinki. They underwent simultaneous bilateral implantation of DBS electrodes in the GPi. The surgical procedure has been described previously (Kupsch et al., 2006; Reese et al., 2011; Brücke et al., 2008b). The DBS electrode used was model 3389 (Medtronic Neurological Division) with four platinum-iridium cylindrical surfaces $(1.27 \mathrm{~mm}$ diameter and $1.5 \mathrm{~mm}$ length) and a contact-to-contact separation of 0.5 $\mathrm{mm}$. Target coordinates were based on direct visualization of the GPi in the individual stereotactic $\mathrm{T}_{2}$-weighted MRI. The intended coordinates at the tip of electrode 0 were $17.4-22 \mathrm{~mm}$ lateral from the midline, 2-4 $\mathrm{mm}$ in front of the midcommissural point, and 2-4 $\mathrm{mm}$ below the midcommissural point as determined by MRI adjusted to the individual patient's anatomy. Correct placement of the DBS electrode was confirmed by intraoperative microelectrode recordings and direct macrostimulation in all patients. Moreover, localization of DBS electrodes was verified by postoperative MRI (Berlin) or stereotactic CT (Hannover) imaging using automated normalization and contact localization in standard Montreal Neurological Institute (MNI) stereotactic space coordinates [methods are described in more detail in the study by Schönecker et al. (2009)] in all patients (except for case 16 and 22 where image quality of postoperative CT was not sufficient). Evaluation of clinical efficacy of chronic DBS on motor symptoms revealed an overall mean improvement of 46.5\% [using TWSTRS (Toronto Western spasmodic torticollis rating scale, case 1-8 and 10-22) and BFMDRS (Burke-Fahn-Marsden dystonia rating scale, case 9) at least 3 months after DBS surgery], which further supported correct placement of DBS electrodes in our patients.

Recordings. LFP recordings were made on average at $3 \pm 1$ (range 1-4) $\mathrm{d}$ postoperatively before chronic stimulation was commenced. GPi LFPs were recorded bipolarly from the four adjacent contacts of each DBS electrode (contact pairs $01,12,23$ ). The bipolar setup was used to ensure a local origin of signals and minimizes the possibility of cortical activity picked up by the electrodes (Kühn et al., 2004). Signals were amplified $(\times 50,000)$ and filtered at $1-250 \mathrm{~Hz}$ using a custom-made, highimpedance amplifier (which had as its front end input stage the INA128 instrumentation amplifier, Texas Instruments Incorporated $12500 \mathrm{TI}$ Boulevard) or a Digitimer D360 amplifier with bandpass filter set at $0.5-500 \mathrm{~Hz}$ and recorded through a $1401 \mathrm{~A}-\mathrm{D}$ converter (Cambridge Electronic Design) onto a computer using Spike2 software (Cambridge Electronic Design; cases 3-5, 8-11, 14-16, 18-22). Signals were sampled at $1 \mathrm{kHz}$ and monitored online. In cases 1, 2, 6, 7, 12, 13, and 17 a different amplifier (Biopotential Analyzer Diana) was used with a sampling rate of $1.5 \mathrm{kHz}$. EMG activity was recorded from upper limb muscles during arm movements using $\mathrm{Ag}-\mathrm{AgCl}$ surface electrodes, filtered at $10-250 \mathrm{~Hz}$ and amplified $(\times 1000)$ and recorded as described above. EEG recordings were obviated by surgical wound dressing in our patients.

Paradigm. LFPs were recorded while patients engaged in a choicereaction time task where forearm pronation movements with three different amplitudes had to be performed (see schematic of paradigm, Fig. $1 \mathrm{~A})$. Patients were seated comfortably in a chair and held a rotatable light handle with one hand. Rotation movements were sensed using a potentiometer and recorded via a $1401 \mathrm{~A}-\mathrm{D}$ converter onto a computer using Spike 2 software. One of three possible cues appeared in randomized order on a laptop screen for $1 \mathrm{~s}$ and with intertrial periods of $4-6 \mathrm{~s}$. Cues indicated the size of the required forearm pronation movements to reach the target position (Fig. 1). The patients were instructed to perform the movement as quickly and accurately as possible. The aim was to reach the indicated target positions with a swift movement of the handle (pronation) and to rotate the handle back to the start position (supination) without delay after reaching the target position. The arm movements differed in the maximal angular amplitude that was necessary to reach the target position: small $\left(\mathrm{S}, 30^{\circ}\right)$, medium $\left(\mathrm{M}, 60^{\circ}\right)$, and large $\left(\mathrm{L}, 90^{\circ}\right) \mathrm{am}$ plitude arm movements. The position of the handle was shown online on the laptop screen as a red dot in relation to the start and target positions in 13 patients (cases $10-22,21$ sides). Patients 1-9 had no such visual feedback. Patients performed the experiment separately with the right and left arm. Eight of the 22 patients performed the task with only one arm due to time constraints or because prominent dystonic laterocollis obviated maintenance of a constant position with which to view the laptop screen while holding the handle of the device. A total of 36 sides were tested. Importantly, patients did not show dystonic features of the hand as judged by clinical examination. The analysis of movementrelated changes in neuronal activity was limited to the initial phase of the movement, i.e., the pronation movement to reach the target position. In addition to the parametric three step increase of movement amplitude, we investigated the relation between $\gamma$ synchronization and movement amplitude/speed on a single trial level with freely chosen movement size within a range from $1^{\circ}$ to $90^{\circ}$ in a single subject (patient 2, left hand movements). Movements were externally paced but no target amplitude was given ( $n=62$ trials).

To explore whether movement-related $\gamma$ band synchronization was sensitive to movement direction, four patients repeated the initial task (with initial pronation movements) with reverse movement direction starting with supination movements (patients $4,7,8$, and $13 ; n=5$ sides). The mean $\gamma$ band activity during the initial movement phase $(0-300 \mathrm{~ms}$ after movement onset) was compared between movements with opposing directions.

To ensure that pallidal $\gamma$ synchronization was related to the active movement rather than induced by proprioceptive afferents during arm movements, we performed passive movements in a subgroup of patients (patients $10-13, n=6$ sides). Here, patients held the handle of the device but tried to keep their arm as relaxed as possible while the handle was moved by the investigator with similar velocity as in the active movement trials. Trials were visually inspected and periods of increased EMG activity during the passive pronation movements were excluded from further analysis.

Analysis. Files with a higher sampling rate were resampled to a common sampling rate of $1 \mathrm{kHz}$. LFP traces were visually checked for artifacts and trials with a reaction time $>1 \mathrm{~s}$ or where the patient executed the wrong movement were rejected, leaving an average of $38 \pm 3$ (S, range 21-64), $39 \pm 3$ (M, range 26-68), and $38 \pm 3$ (L, range 21-68) trials per movement condition per side for further analysis (no significant difference between movement conditions).

Movement-related changes in neuronal synchronization were calculated in trials of $6 \mathrm{~s}$ duration and expressed as percentage change from baseline activity (calculated from -2 to $-1 \mathrm{~s}$ before movement onset) separately for each movement amplitude. To better separate LFP oscillatory changes related to movement amplitude or speed, LFP traces were aligned to three different time points: (1) movement onset (MO), (2) maximum velocity per trial $(\operatorname{maxV})$, and $(3)$ the time point of reaching the target position, i.e., maximum amplitude ( $\operatorname{maxA}$ ). Spectra were estimated using the sliding window discrete Fourier transform in Spike2 with a window size of 512 data points (centered on time 0 assigned 
Table 1. Patient details

\begin{tabular}{|c|c|c|c|c|c|c|c|c|c|}
\hline $\begin{array}{l}\text { Patient } \\
\text { ID }\end{array}$ & $\begin{array}{l}\text { Surgical } \\
\text { center }\end{array}$ & $\begin{array}{l}\text { Age/ } \\
\text { Gender }\end{array}$ & $\begin{array}{l}\text { Disease } \\
\text { duration } \\
\text { in years }\end{array}$ & $\begin{array}{l}\text { Disease/Affected } \\
\text { body part }\end{array}$ & $\begin{array}{l}\text { Clinical scores } \\
\text { pre0P (TWSTRS } \\
\text { severity subscore) }\end{array}$ & $\begin{array}{l}\text { Clinical scores } \\
\text { postOP (TWSTRS } \\
\text { severity subscore) }\end{array}$ & Medication & $\begin{array}{l}\text { Selected } \\
\text { contact }\end{array}$ & Stimulation settings \\
\hline 1 & Hannover & $45 / \mathrm{M}$ & 8 & Cervical dystonia & 20 & 8 & Trihexiphenidyl $8 \mathrm{mg} / \mathrm{d}$ & $\mathrm{R} 12 \mathrm{~L} 12$ & $\begin{array}{l}\mathrm{R}: 1-, 2+; 210 \mu \mathrm{s}, 130 \mathrm{~Hz}, 5.2 \mathrm{~V} \\
\mathrm{~L}: 1-, 2+; 210 \mu \mathrm{s}, 130 \mathrm{~Hz}, 5.2 \mathrm{~V}\end{array}$ \\
\hline 2 & Hannover & $64 / M$ & 4 & $\begin{array}{l}\text { Segmental dystonia } \\
\text { (neck, mouth) }\end{array}$ & 21 & 8 & Lorazepam $2 \mathrm{mg} / \mathrm{d}$ & R12 L01 & $\begin{array}{l}\mathrm{R}: 1-, 2+; 210 \mu \mathrm{s}, 130 \mathrm{~Hz}, 3.2 \mathrm{~V} \\
\mathrm{~L}: 1-, 2+; 210 \mu \mathrm{s}, 130 \mathrm{~Hz}, 3.32 \mathrm{~V}\end{array}$ \\
\hline 3 & Berlin & $57 / \mathrm{F}$ & 8 & Cervical dystonia & 23 & 10 & Clonazepam $2 \mathrm{mg} / \mathrm{d}$ & $\mathrm{R} 12$ & $\begin{array}{l}\mathrm{R}: 1-, 2-90 \mu \mathrm{s}, 180 \mathrm{~Hz}, 2.2 \mathrm{~V} \\
\mathrm{~L}: 1-, 2-; 90 \mu \mathrm{s}, 180 \mathrm{~Hz}, 3 \mathrm{~V}\end{array}$ \\
\hline 4 & Berlin & $50 / \mathrm{F}$ & 9 & Cervical dystonia & 28 & 4 & None & R23 L01 & $\begin{array}{l}\mathrm{R}: 2-, 3-; 90 \mu \mathrm{s}, 130 \mathrm{~Hz}, 3,3 \mathrm{~V} \\
\mathrm{~L}: 2-, 3-; 90 \mu \mathrm{s}, 130 \mathrm{~Hz}, 3,5 \mathrm{~V}\end{array}$ \\
\hline 5 & Berlin & $56 / \mathrm{F}$ & 9 & Cervical dystonia & 16 & 17 & $\begin{array}{c}\text { Trihexyphenidyl } 14 \text { mg/d, } \\
\text { Mirtazapine } 15 \mathrm{mg} / \mathrm{d}, \\
\text { Tetrazepam } 200 \mathrm{mg} / \mathrm{d}\end{array}$ & R01 L12 & OFF/ no treatment response \\
\hline 6 & Hannover & $50 / \mathrm{F}$ & 8 & Cervical dystonia & 23 & 10 & Clonazepam $2 \mathrm{mg} / \mathrm{d}$ & $\mathrm{R} 23$ & $\begin{array}{l}\mathrm{R}: 1-, 2+; 210 \mu \mathrm{s}, 130 \mathrm{~Hz}, 3,8 \text { Volt } \\
\mathrm{L}: 1-, 2+; 210 \mu \mathrm{s}, 130 \mathrm{~Hz}, 3.8 \text { Volt }\end{array}$ \\
\hline 7 & Berlin & $56 / \mathrm{F}$ & 4 & Cervical dystonia & 26 & 10 & Trihexyphenidyl 15 mg/d & R01 L12 & $\begin{array}{l}\mathrm{R}: 1-, 90 \mu \mathrm{s}, 180 \mathrm{~Hz}, 3.7 \mathrm{~V} \\
\mathrm{~L}: 2-; 90 \mu \mathrm{s}, 180 \mathrm{~Hz}, 3.7 \mathrm{~V}\end{array}$ \\
\hline 8 & Berlin & $41 / \mathrm{M}$ & 9 & Cervical dystonia & 23 & 11 & None & $\mathrm{L} 12$ & $\begin{array}{l}\mathrm{R}: 2-, 120 \mu \mathrm{s}, 130 \mathrm{~Hz}, 4.5 \mathrm{~V} \\
\mathrm{~L}: 2-; 120 \mu \mathrm{s}, 130 \mathrm{~Hz}, 3.3 \mathrm{~V}\end{array}$ \\
\hline 9 & Berlin & $57 / \mathrm{F}$ & 2 & Focal dystonia (mouth) & $\begin{array}{c}4 / 40^{a} \\
12 / 120^{b}\end{array}$ & $\begin{array}{c}3 / 40^{a} \\
10 / 120^{b}\end{array}$ & None & R01 L23 & $\begin{array}{l}\mathrm{R}: 1-, 2-, 90 \mu \mathrm{s}, 130 \mathrm{~Hz}, 2.1 \mathrm{~V} \\
\mathrm{~L}: 1-, 2-; 90 \mu \mathrm{s}, 130 \mathrm{~Hz}, 2.2 \mathrm{~V}\end{array}$ \\
\hline 10 & Berlin & $51 / \mathrm{M}$ & 15 & Cervical dystonia & 23 & 18 & $\begin{array}{l}\text { Lithium } 900 \mathrm{mg} / \mathrm{d}, \\
\text { Escitalopram } 10 \mathrm{mg} / \mathrm{d}\end{array}$ & R12 L12 & $\begin{array}{l}\mathrm{R}: 3-, 90 \mu \mathrm{s}, 130 \mathrm{~Hz}, 6 \mathrm{~V} \\
\mathrm{~L}: 3-; 90 \mu \mathrm{s}, 130 \mathrm{~Hz}, 6 \mathrm{~V}\end{array}$ \\
\hline 11 & Hannover & $38 / \mathrm{M}$ & 23 & Segmentale dystonia & 25 & 12 & None & $\mathrm{L} 12$ & $\begin{array}{l}\mathrm{R}: 1-, 2+; 210 \mu \mathrm{s}, 130 \mathrm{~Hz}, 2.4 \mathrm{~V} \\
\mathrm{~L}: 1-, 2+; 210 \mu \mathrm{s}, 130 \mathrm{~Hz}, 1.8 \mathrm{~V}\end{array}$ \\
\hline 12 & Hannover & $49 / M$ & 12 & Cervical dystonia & 15 & 8 & Clonazepam $2 \mathrm{mg} / \mathrm{d}$ & L01 & $\begin{array}{l}\text { R: } 1-, 2+; 210 \mu s, 130 \mathrm{~Hz}, 3,2 \text { Volt } \\
\text { L: } 1-, 2+; 210 \mu \mathrm{s}, 130 \mathrm{~Hz}, 3.2 \text { Volt }\end{array}$ \\
\hline 13 & Hannover & $46 / \mathrm{M}$ & 5 & Cervical dystonia & 24 & 16 & Citalopram $20 \mathrm{mg} / \mathrm{d}$ & R01 L12 & $\begin{array}{l}\mathrm{R}: 1-, 2+; 120 \mu \mathrm{s}, 130 \mathrm{~Hz}, 5.3 \mathrm{~V} \\
\mathrm{~L}: 1-, 2+; 120 \mu \mathrm{s}, 130 \mathrm{~Hz}, 5.3 \mathrm{~V}\end{array}$ \\
\hline 14 & Berlin & $57 / \mathrm{F}$ & 7 & $\begin{array}{l}\text { Segmental dystonia } \\
\text { (neck, mouth) }\end{array}$ & 23 & 10 & None & L01 & $\begin{array}{l}\mathrm{R}: 0-, 1-; 90 \mu \mathrm{s}, 180 \mathrm{~Hz}, 2.6 \mathrm{~V} \\
\mathrm{~L}: 0-, 1-; 90 \mu \mathrm{s}, 180 \mathrm{~Hz}, 2.6 \mathrm{~V}\end{array}$ \\
\hline 15 & Berlin & $55 / \mathrm{M}$ & 8 & Cervical dystonia & 25 & 12 & None & R23 L23 & $\begin{array}{l}\mathrm{R}: 1-, 2-; 90 \mu \mathrm{s}, 135 \mathrm{~Hz}, 2.1 \mathrm{~V} \\
\mathrm{~L}: 1-, 2-; 90 \mu \mathrm{s}, 130 \mathrm{~Hz}, 2.1 \mathrm{~V}\end{array}$ \\
\hline 16 & Hannover & $57 / M$ & 21 & Cervical dystonia & 27 & 20 & Clonazepam $2 \times 1 \mathrm{mg}$ & R23 L01 & $\begin{array}{l}\mathrm{R}: 1-, 2+; 180 \mu \mathrm{s}, 130 \mathrm{~Hz}, 3.5 \mathrm{~V} \\
\mathrm{~L}: 1-, 2+; 180 \mu \mathrm{s}, 130 \mathrm{~Hz}, 3.6 \mathrm{~V}\end{array}$ \\
\hline 17 & Hannover & $66 / M$ & 17 & Cervical dystonia & 19 & 8 & $\begin{array}{l}\text { Lorazepam } 2 \mathrm{mg} / \mathrm{d} \\
\text { Baclofen } 100 \mathrm{mg} / \mathrm{d}\end{array}$ & $\mathrm{L} 12$ & $\begin{array}{l}\mathrm{R}: 1-, 2+; 210 \mu \mathrm{s}, 130 \mathrm{~Hz}, 1.7 \mathrm{~V} \\
\mathrm{~L}: 1-, 2+; 210 \mu \mathrm{s}, 130 \mathrm{~Hz}, 2 \mathrm{~V}\end{array}$ \\
\hline 18 & Berlin & $53 / \mathrm{F}$ & 3 & Cervical dystonia & 23 & 9 & $\begin{array}{l}\text { Telmisartan } 40 \mathrm{mg} / \mathrm{d}, \\
\text { Hydrochlorothiazid } \\
12.5 \mathrm{mg} / \mathrm{d}\end{array}$ & $\mathrm{L} 23$ & $\begin{array}{l}\mathrm{R}: 1-; 90 \mu \mathrm{s}, 140 \mathrm{~Hz}, 3 \mathrm{~V} \\
\mathrm{~L}: 2-; 90 \mu \mathrm{s}, 140 \mathrm{~Hz}, 3.5 \mathrm{~V}\end{array}$ \\
\hline 19 & Berlin & $43 / \mathrm{F}$ & 8 & Cervical dystonia & 18 & 10 & Tetrazepam $50 \mathrm{mg} / \mathrm{d}$ & R12 L01 & $\begin{array}{l}\mathrm{R}: 1-; 60 \mu \mathrm{s}, 130 \mathrm{~Hz}, 2.8 \mathrm{~V} \\
\mathrm{~L}: 1-; 60 \mu \mathrm{s}, 130 \mathrm{~Hz}, 2.8 \mathrm{~V}\end{array}$ \\
\hline 20 & Berlin & $33 / \mathrm{M}$ & 2 & Cervical dystonia & 23 & 14 & None & R01 L12 & $\begin{array}{l}\mathrm{R}: 2-, 3-; 90 \mu \mathrm{s}, 130 \mathrm{~Hz}, 2.3 \mathrm{Volt} \\
\mathrm{L}: 2-, 3-; 90 \mu \mathrm{s}, 130 \mathrm{~Hz} \\
1.5 \text { Volt }\end{array}$ \\
\hline 21 & Berlin & $49 / \mathrm{F}$ & 7 & Cervical dystonia & 20 & 8 & None & $\mathrm{R} 12 \mathrm{~L} 12$ & $\begin{array}{l}\mathrm{R}: 2-; 90 \mu \mathrm{s}, 130 \mathrm{~Hz}, 1.7 \mathrm{~V} \\
\mathrm{~L}: 2-; 90 \mu \mathrm{s}, 130 \mathrm{~Hz}, 3.1 \mathrm{~V}\end{array}$ \\
\hline 22 & Hannover & $67 / \mathrm{F}$ & 5 & Cervical dystonia & 14 & 5 & $\mathrm{~L}-$ Thyroxin $75 \mu \mathrm{g} / \mathrm{d}$ & R12 L12 & $\begin{array}{l}\mathrm{R}: 1-, 2+; 210 \mu \mathrm{s}, 130 \mathrm{~Hz}, 3 \mathrm{~V} \\
\mathrm{~L}: 1-, 2+; 210 \mu \mathrm{s}, 130 \mathrm{~Hz}, 3 \mathrm{~V}\end{array}$ \\
\hline Mean $( \pm S D)$ & & $\begin{array}{l}52( \pm 8) \\
\quad 11 \mathrm{~F}, 11 \mathrm{M}\end{array}$ & $8.8( \pm 5.6)$ & & $21.9( \pm 3.8)$ & $10.9( \pm 4.1)$ & & & \\
\hline
\end{tabular}

M, Male; F, female; $R$, right; L, left.

${ }^{a}$ AIMS (Abnormal involuntary movement scale).

${ }^{b}$ Burke-Fahn-Marsden dystonia rating scale, movement subscale.

time $=0)$ and shifted in $100 \mathrm{~ms}$ steps across epochs. A Hanning window was applied to attenuate edge effects. The frequency resolution of spectra was $1.95 \mathrm{~Hz}$. LFPs were analyzed over frequencies between 1 and $200 \mathrm{~Hz}$. Time-frequency plots were calculated showing median percentage power changes relative to baseline for pallidal LFPs averaged over all contact pairs per electrode, separately for the contralateral and ipsilateral side.

According to the main oscillatory changes seen in the grand average from all patients and in line with the results of previous studies (Androu- lidakis et al., 2007; Brücke et al., 2008a,b), we determined specific time periods and frequency bands of interest ( $\beta$-band: $20-30 \mathrm{~Hz}$ from -300 $\mathrm{ms}$ to $300 \mathrm{~ms}$ around MO; $\gamma$-band: $60-80 \mathrm{~Hz}$ from MO to $300 \mathrm{~ms}$ after $\mathrm{MO}$ ) for the main analysis. Since there were no task-specific changes in oscillatory activity in pallidal recordings ipsilateral to the moved side, all further analysis was limited to the contralateral GPi. Furthermore, the contact pair that displayed the largest power changes compared with baseline activity in the region of interest averaged over the three movement conditions was selected for all further analysis. Contact evaluation 
A

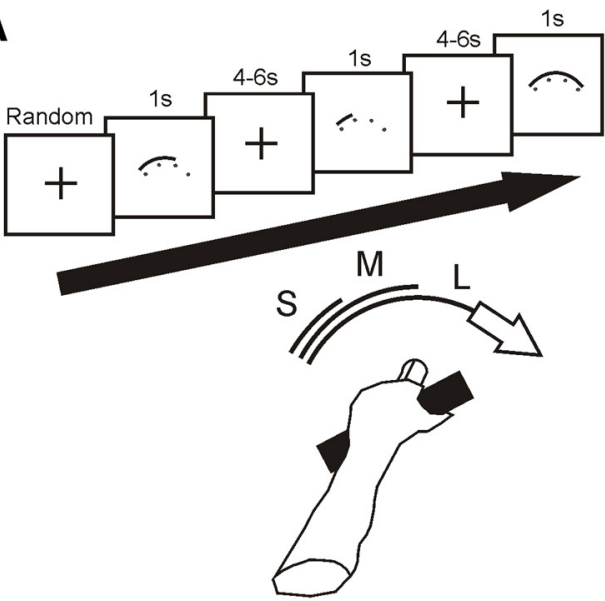

C

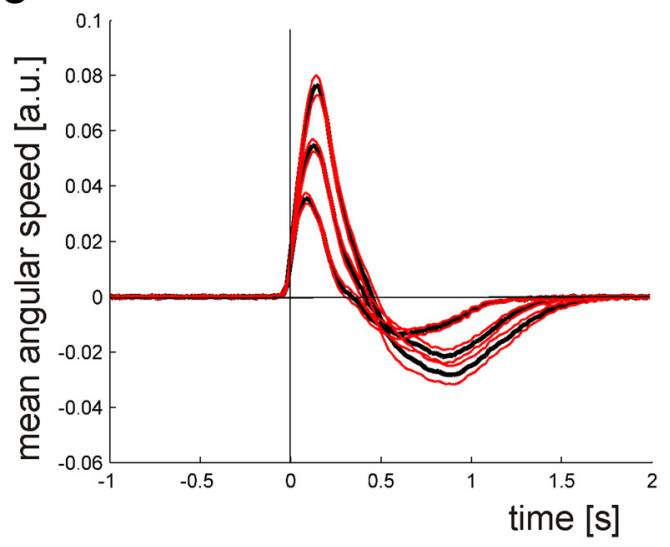

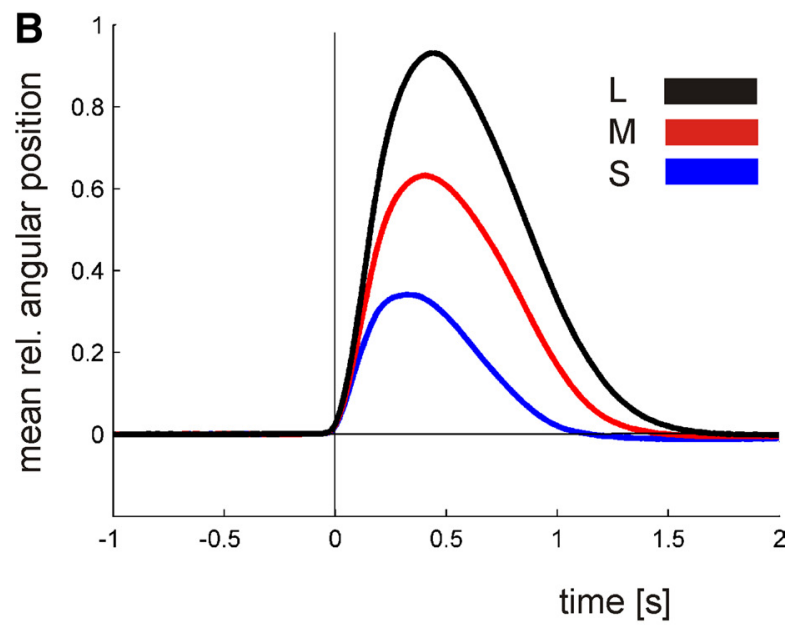

D

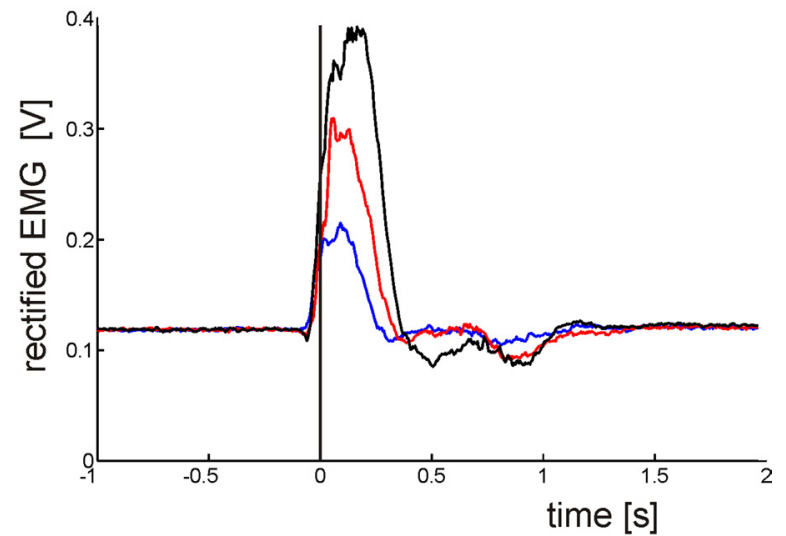

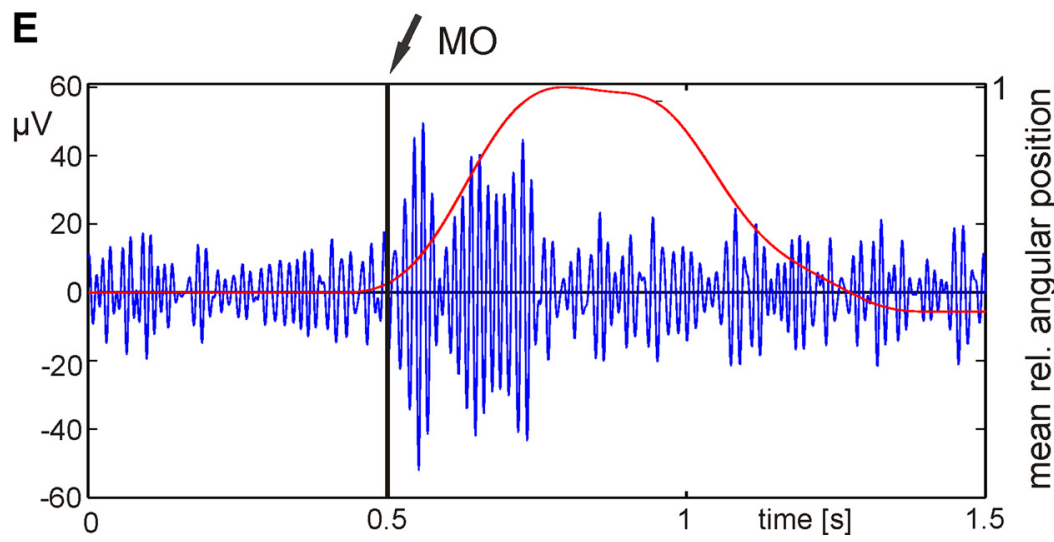

Figure 1. $\quad \boldsymbol{A}$, Schematic illustration of the paradigm. $\boldsymbol{B}$, Mean relative angular movement positions averaged across sides (small amplitude movement in dark blue, medium amplitude movements in red, and large amplitude movements in black). C, Mean angular speed averaged across sides separately for the three conditions (mean \pm SE). $\boldsymbol{D}$, Mean rectified EMG activity from the forearm of patient 4. E, Trace of $55-90 \mathrm{~Hz}$ filtered LFP during a movement with large amplitude (blue) and angular movement position (red) from patient 7.

revealed that at least one of the contacts from each bipolar recording used for the main analysis lay within GPi on postoperative imaging (not known for case 16 and 22 where postoperative imaging data were not available; Fig. 2). In case 2, single trial analysis was performed on filtered $\gamma$ band activity $(60-80 \mathrm{~Hz})$ and movement speed and amplitude of each trial were correlated with the $\gamma$ band response $300 \mathrm{~ms}$ around MO, maxA, and maxV.

To define distinct peaks in the power spectrum, we calculated Fast Fourier Transform (FFT)-based power spectra using $1 \mathrm{~Hz}$ frequency resolution on one rest-recording data segment (55-200 s) in each patient.

Statistics. Since movement related oscillatory activity was not normally distributed between patients in all time-frequency coordinates as as- sessed by the Kolmogorov-Smirnov test (using the command "kstest" from the Matlab statistics toolbox, The MathWorks), movement-related power changes across all patients were analyzed by means of the nonparametric Wilcoxon's signed rank test (command "signrank," statistics toolbox) and Friedman's test of related samples (command "friedman"). In each time-frequency bin of the median time-frequency plot we tested whether changes in contralateral and ipsilateral GPi during arm movements were different from baseline, and compared the time-frequency bins between the three movement conditions and between ipsilateral and contralateral sides. This provided matrices with each bin represented by its respective $p$ value. To correct for multiple comparisons in timefrequency analysis, the false discovery rate (FDR) procedure was used 

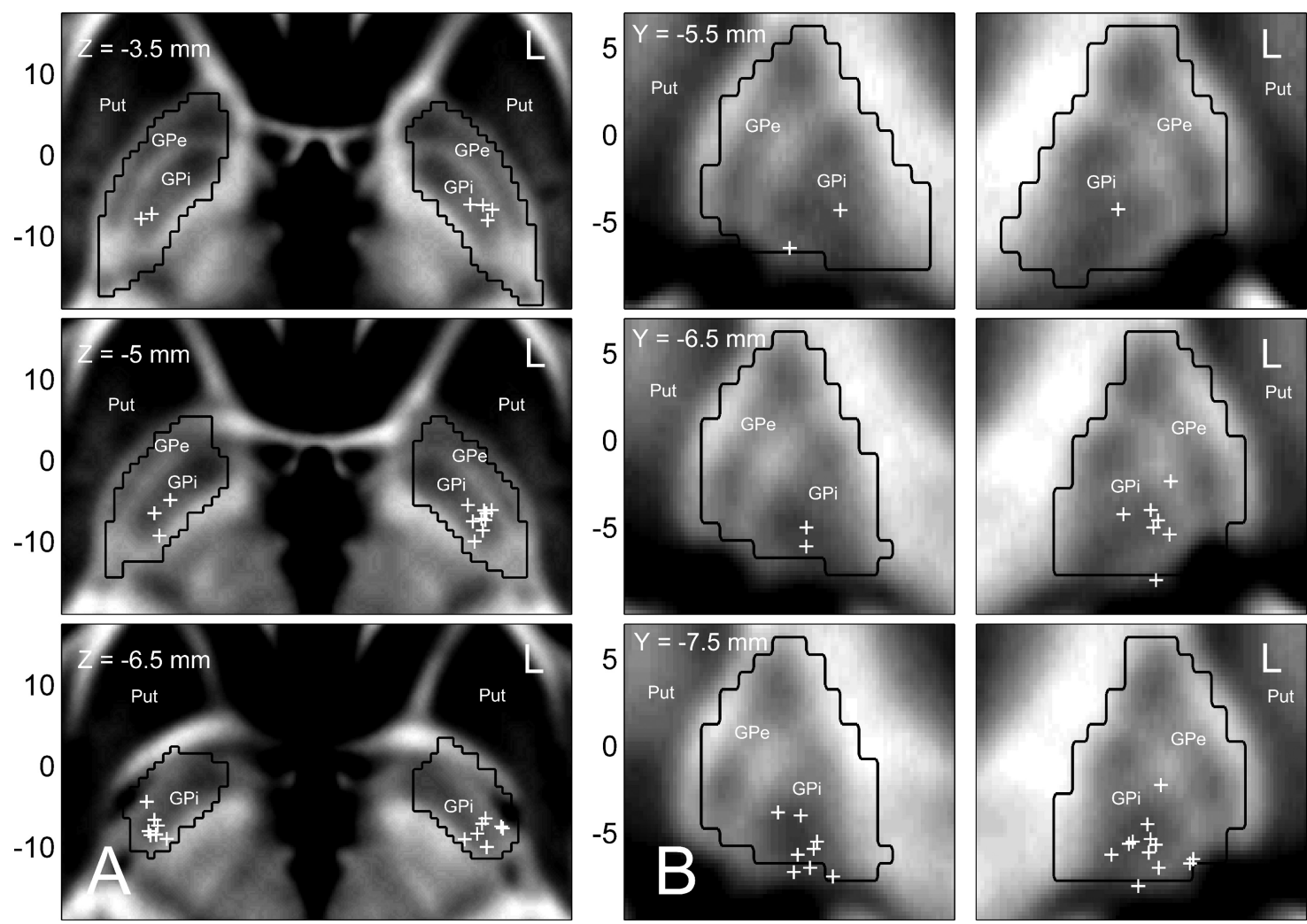

Figure 2. Localizations of contact pairs of the DBS electrodes in standard MNI stereotactic space coordinates (dimensions in mm:X: mediolateral, Y: anteroposterior, Z: dorsoventral direction). $\boldsymbol{A}$, $\boldsymbol{B}$, Localizations of the center $(+)$ of the bipolar contact pairs selected for LFP analysis are depicted (white crosses; $L$, left side) on corresponding horizontal slices $(\boldsymbol{A})$ and coronal slices $(\boldsymbol{B})$ of the MNI standard brain template with the structures: putamen (put), globus pallidum ext. (gpe), globus pallidum int. (gpi). In relation to the center point of the anterior commissure, the horizontal level $z=$ $-3.5 \mathrm{~mm}$ is located $\sim 0.8 \mathrm{~mm}$ more dorsal and the level $z=-5 \mathrm{~mm}$ is located $0.7 \mathrm{~mm}$ more ventral. Mean nuclear boundaries of the globus pallidus are outlined (black) based on the Harvard-0xford subcortical probabilistic structural atlas ( $n=20$ patients). Note that the actual contacts are located up to $1.75 \mathrm{~mm}$ more dorsal (proximal contact) and more ventral (distal contact) with respect to the displayed center of the contact pairs.

and the thresholds corresponding to the $\alpha$ level of $p<0.01$ were calculated (Benjamini and Hochberg, 1995).

For the subsequent analysis, looking at the effects of movement parameters on neuronal activity in selected frequency bands and time-windows (60-80 Hz: $0-300 \mathrm{~ms}$ after MO; 20-30 Hz: -300 to $300 \mathrm{~ms}$ after MO), changes from baseline power were normally distributed according to Kolmogorov-Smirnov test and therefore assessed by repeated-measures ANOVA (PASWStatistics 18, SPSS) with factor MOVEMENT CONDITION (3 levels: $\mathrm{S}, \mathrm{M}$, and $\mathrm{L}$ ) tested separately for power changes related to $\mathrm{MO}$, $\operatorname{maxV}$, and maxA. Subgroup analysis controlling for modifications in online feedback of the movement was performed using the between-subject factor FEEDBACK. Post hoc Student's $t$ tests were performed to determine relevant differences in event-related power changes, and results were corrected for multiple comparisons using Bonferroni corrections. All behavioral parameters (movement speed, amplitude, and reaction time) were assessed using repeated-measures ANOVA with the factors MOVEMENT CONDITION.

The mean relative change in movement velocity between conditions was correlated with the mean relative modulation in $\gamma$ band activity across subjects (Pearson's correlation). Additionally, single-trial analysis was performed in a single subject to evaluate the correlation between the average root-mean square amplitude of the $60-80 \mathrm{~Hz}$ filtered LFP traces from each trial averaged $300 \mathrm{~ms}$ around $\mathrm{MO}, \operatorname{maxV}$, and $\max \mathrm{A}$ and the movement speed of the corresponding trial using Pearson's correlation. All results are reported as mean \pm SEM, unless stated otherwise.

\section{Results}

Behavioral data

Patients performed the task with a mean reaction time of $0.49 \pm$ $0.03 \mathrm{~s}$. The three conditions differed in amplitude and angular velocity of the arm movements (Fig. $1 B-D$; repeated-measures ANOVA
$F_{(1.7)}=4620 ; p<0.001, F_{(1.2)}=230.6 ; p<0.001$, Huyhn-Feldt corrected, respectively). This was the same whether or not visual feedback of the ongoing movement was given online. The target position was reached at different time points with respect to movement amplitude (S: $0.40 \pm 0.02 \mathrm{~s} ; \mathrm{M}: 0.50 \pm 0.02 \mathrm{~s} ; \mathrm{L}: 0.54 \pm 0.02 \mathrm{~s}$ ). Subgroup analysis revealed that movement speed was significantly slower for large movements in those patients that performed the task with online visual feedback compared with those without direct visual feedback (repeated-measures ANOVA interaction between MOVEMENT CONDITION and FEEDBACK, $F_{(2)}=33 ; p<0.001$, post hoc two-sided Student's $t$ test: $p=0.027$, Bonferroni corrected).

\section{General pattern of movement-related changes in oscillatory activity}

Without prior selection of the pallidal recording sites, the time frequency plots averaged across all bipolar recordings in all patients ( $n=22$ patients, 36 hemispheres, total of 104 recording sites) contralateral to the moved side revealed a significant eventrelated desynchronization (ERD) in the $\beta$ frequency range $(20-30 \mathrm{~Hz})$ starting before movement onset and an $\alpha(8-12 \mathrm{~Hz})$ ERD during movement, as well as a broad event-related synchronization (ERS) in the $\gamma$ frequency band from $\sim 35-105 \mathrm{~Hz}$ in all three movement conditions (Fig. $3 A-C$ ). Also, the $\beta$ ERD was followed by a rebound synchronization ( $\beta$ ERS) after the end of the movement. Ipsilateral pallidal LFPs showed a similar ERD in the $\alpha$ and $\beta$ frequency range but a significantly smaller $\gamma$ band ERS compared with the contralateral side for all three movement 


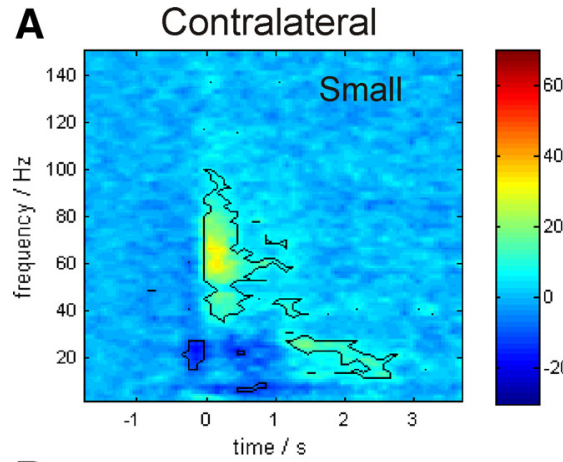

B

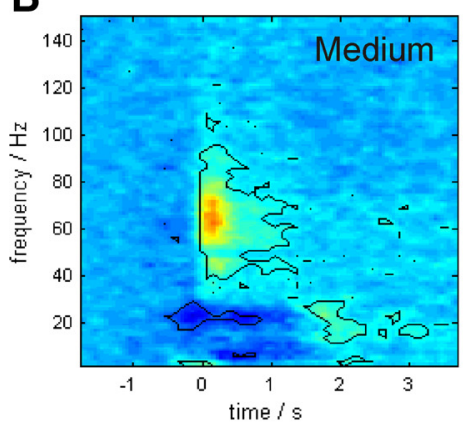

C
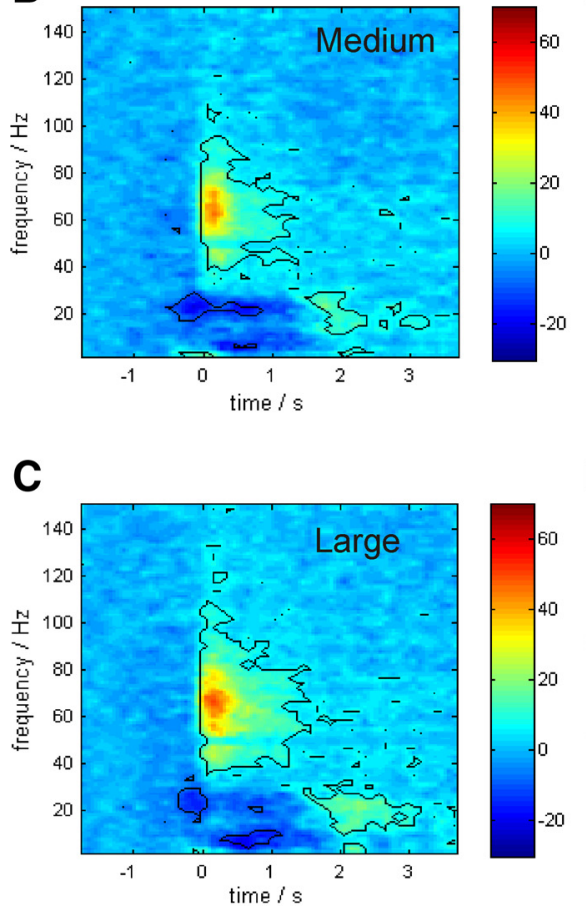

D

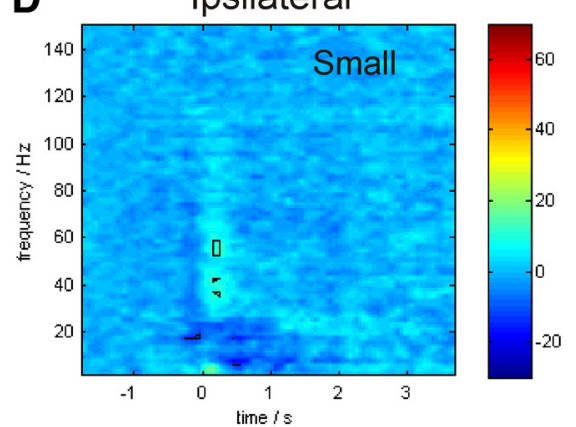

E

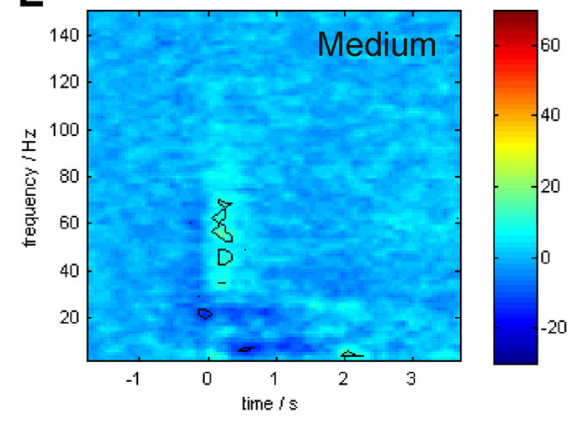

$\mathbf{F}$

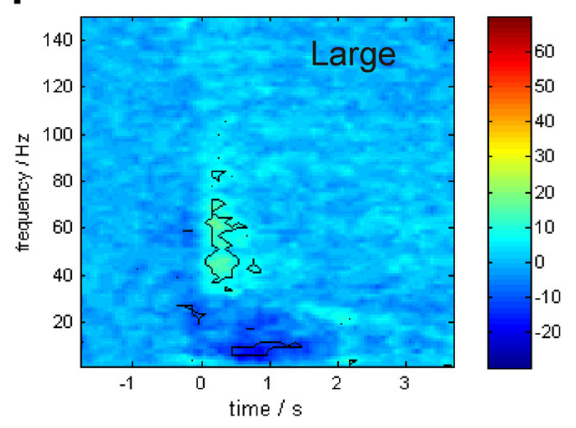

G

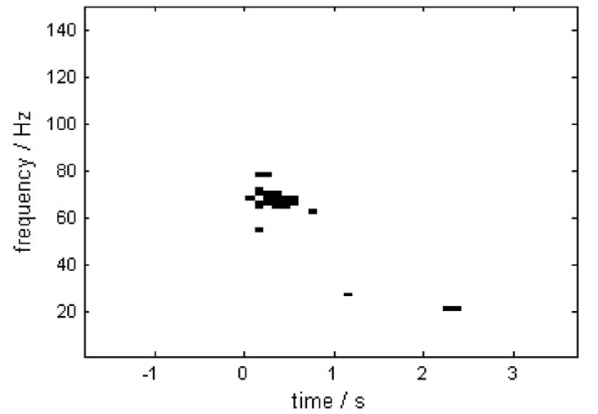

$\mathbf{H}$
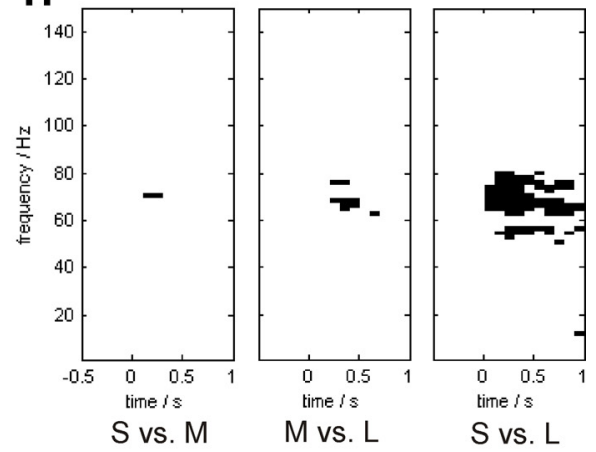

Figure 3. $\boldsymbol{A}-\boldsymbol{F}$, Median oscillatory power changes of 22 patients, 36 contralateral $(\boldsymbol{A}-\boldsymbol{C})$ and ipsilateral $(\boldsymbol{D}-\boldsymbol{F}) \mathrm{GPi}$ and three contact pairs per electrode $(n=104$ per side) during small $(\boldsymbol{A}, \boldsymbol{D})$, medium $(\boldsymbol{B}, \boldsymbol{E})$, and large $(\boldsymbol{C}, \boldsymbol{F})$ amplitude movements. Data are expressed as percentage power change compared with baseline $(-2$ to $-1 \mathrm{~s}$ before movement onset at time point 0$)$. Contour lines surround bins with $p<0.01$ (Wilcoxon's signed rank test, FDR corrected). $\mathbf{G}$, Friedman's test of related samples showing bins $p<0.01$, FDR corrected between movement conditions for contralateral GPi. $\boldsymbol{H}$, Post hoc Wilcoxon's test of oscillatory power changes between movement conditions for contralateral GPi with bins $p<0.01$, FDR corrected.

conditions (see Fig. 3D--F). This pattern was similar to previous findings described in pallidal recordings in patients with dystonia (Brücke et al., 2008b) as well as PD patients on levodopa treatment (Androulidakis et al., 2007).

The contralateral $\gamma$ ERS had a peak reactivity at $\sim 70 \mathrm{~Hz}$ and started $\sim 100$ ms before movement onset and persisted for up to $\sim 600 \mathrm{~ms}$ for the smallest and $\sim 1.5 \mathrm{~s}$ for the largest movement. More importantly, the $\gamma$ ERS showed a parametric modulation with movement amplitudes. Figure $3 G$ shows the bins in the time-frequency plot with a significant difference in median oscillatory power between the three movement conditions (Friedman's test of related samples; $p<0.01$, FDR corrected). The significant bins were restricted to the $\gamma$ frequency range around the peak reactivity $\sim 70 \mathrm{~Hz}$ and started $\sim 100 \mathrm{~ms}$ after MO. Post hoc Wilcoxon's signed rank test confirms the respective differences of median oscillatory power between the three movement conditions focused at $\sim 70 \mathrm{~Hz}$ (Fig. $3 \mathrm{H}$ ). No difference in movement-related oscillatory power changes was revealed between the three movement conditions in the lower frequency range ( $\alpha$ and $\beta$ band) or on the ipsilateral side (data not shown).
Increases in $\gamma$ band activity were restricted to movement-related LFP activity. No distinct $\gamma$ peak was observed in the power spectra from rest recordings.

\section{Selection of frequency bands and contact pairs}

Based on the main findings in the grand average and previous reports, we selected two frequency bands for further analysis that captured the main features of oscillatory power changes during movement. Based on the median matrices, these bands were empirically defined as $20-30 \mathrm{~Hz}$ ( $\beta$ band) and $60-80 \mathrm{~Hz}$ ( $\gamma$ band). The contact pair per electrode that showed the strongest reactivity in those frequency bands before and during movement was selected for further analysis. We found a well defined maximum of $\beta$ and $\gamma$ power changes at one of the contact pairs of the DBS-electrode in all patients, suggesting a locally generated activity. The mean reduction in $\beta$ ERD at the remaining contact pairs was $44.3 \pm 5.9 \%$ ( $p<0.05$, two-sided Student's $t$ test $)$. Similarly, the largest $\gamma$ ERS was $43.7 \pm 8.3 \%$ higher $(p<0.001$, two-sided Student's $t$ test) compared with the average of the remaining contact pairs from the same electrode. The correlation of 
LFP waveforms between contact pairs revealed a phase reversal in 22 of 36 sides. Electrode placement was verified on postoperative imaging using automated normalization and contact localization in standard MNI stereotactic space coordinates in all patients. In this way we could confirm that all contacts used in our main analysis lay within GPi in all patients (except for case 16 and 22 where image quality of postoperative CT was not sufficient; Fig. $2)$. Furthermore, in 30 of the 36 selected best contact pairs $(83.3 \%)$ that showed the highest $\gamma$ ERS during movement, at least one contact was also used during chronic DBS in our patients, which further supports electrode localization in the motor region of the pallidum.

Movement-related pallidal activity and movement kinematics Time-evolving power changes for the selected frequency bands normalized to baseline activity revealed a significant $\gamma$ ERS (Fig. $4 A$ ) and $\beta$ ERD (Fig. $4 B$ ). The $\gamma$ ERS started $\sim 100 \mathrm{~ms}$ before movement onset and lasted up to $\sim 1.5 \mathrm{~s}$ after movement onset (Fig. $4 A$ ), although the limited temporal resolution resulting from smoothing has to be considered in our data. Significant departures of the $\beta$ ERD from baseline started before movement onset and were ongoing during movement. A significant parametric modulation of oscillatory power changes with movement amplitude only occurred in the $60-80 \mathrm{~Hz}$ band. Direct comparison of power changes using time-evolving Wilcoxon's signed rank test revealed significant differences between all movement conditions in the $60-80 \mathrm{~Hz}$ band (Fig. $4 A, p<0.05$, FDR corrected). Here, the $\gamma$ ERS was strongest during movements with the largest amplitude and the weakest $\gamma$ ERS occurred with small movements. In contrast, no significant difference in $\beta$ ERD occurred with respect to movement amplitude before, during, and up to one second after movement (Fig. $4 B$ ).

These features were confirmed by repeated-measures ANOVAs with the main factor MOVEMENT CONDITION (3 levels: small, medium, large) for $\gamma$ band activity averaged in the time-window from MO to $300 \mathrm{~ms}$ after MO (Fig. 5A). We observed a main effect for movement condition $\left(F_{(1.2)}=17.6\right.$; $p<0.001$; Huyhn-Feldt corrected), and post hoc Student's $t$ test revealed a significant difference between all conditions with a mean $\gamma$ ERS of $57.6 \pm 9 \%$ for small, $82.0 \pm 12.2 \%$ for medium, and $105.7 \pm 17.6 \%$ for large movements (small vs medium: $p<0.001$, small vs large: $p<0.001$, and medium vs large $p=0.015$, Bonferroni corrected). Whether or not patients had direct online visual feedback during task performance had no effect on the main result as shown by the ANOVA using feedback as between-subject factor $\left(F_{(1)}=2.26 ; p=0.142\right)$. Accordingly, the main results of movement-related increase in $\gamma$ ERS could be equally shown in both subgroups $\left[F_{(1.1)}=12.3 ; p=\right.$ 0.02 (Huyhn-Feldt corrected) and $F_{(2)}=11 ; p<0.001$, respectively]. On an individual level, the movement-related increase in $\gamma$ ERS was found in 92 of 106 recording sites.

In contrast, for $\beta$ band activity (Fig. $5 B$ ), the repeated-measures ANOVA with the main factor MOVEMENT CONDITION (3 levels: small, medium, large) and TIME [2 levels: preMO ( $-300 \mathrm{~ms}$ to $\mathrm{MO}$ ) vs postMO (MO to $300 \mathrm{~ms}$ )] showed no significant effect (MOVEMENT CONDITION: $F_{(2)}=0.6 ; \mathrm{p}=0.52$ and TIME: $\left.F_{(1)}=0.5 ; \mathrm{p}=0.48\right)$ or interaction $\left(F_{(2)}=3 ; \mathrm{p}=0.053\right)$. Additionally, we conducted a separate ANOVA for $\beta$-ERD in the premovement time period that did not reveal a significant effect for condition.

Our main analysis revealed a stepwise increase in eventrelated power changes with increasing amplitude of the movement that was specific to $\gamma$ band activity. To further delineate a potential role of pallidal activity in parameterization of move- ments, we set out to more specifically evaluate oscillatory changes in $\gamma$ band activity with regard to movement direction, amplitude, and velocity as well as proprioceptive feedback. These analyses were restricted to changes in the $60-80 \mathrm{~Hz}$ band based on our general findings.

\section{Role of movement direction}

In a subgroup of patients $(n=4$ patients, 5 sides, 14 contact pairs), we tested whether movement-related $\gamma$ band synchronization was sensitive to movement direction. The mean $\gamma$ ERS in the time-period from MO to $300 \mathrm{~ms}$ after MO was similar for both movements with opposite directions: with initial pronation movements (small $44.7 \pm 7.9 \%$, medium $63.8 \pm 10.8 \%$, large $84.7 \pm 14.1 \%)$ and with initial supination movements (small $48.4 \pm 10.8 \%$, medium $64.4 \pm 16.3 \%$, large $104.3 \pm 26.4 \%$; Fig. $6)$. The repeated-measures ANOVA revealed an effect for MOVEMENT AMPLITUDE $\left(F_{(1)}=5.9, \mathrm{p}=0.019\right)$ but no effect of MOVEMENT DIRECTION $\left(F_{(1)}=0.15, \mathrm{p}=0.7\right)$ and no interaction between movement amplitude and movement direction $\left(F_{(1)}=0.7, \mathrm{p}=0.4\right)$. Additional subgroup analysis restricted to movements with initial supination confirmed a significant effect for MOVEMENT AMPLITUDE (ANOVA $F_{(1)}=11.3, p=$ 0.004, Huyhn-Feldt corrected). Post hoc two-sided Student's $t$ test confirmed differences in $\gamma$ ERS according to movement amplitude regardless of the direction of the movement [small vs medium: $p=0.09$ (n.s. after Bonferroni correction), small vs large: $p=0.009$, and medium vs large $p=0.015$, Bonferroni corrected; data not shown]. With respect to the $\gamma$ ERS at the selected best contact pair we defined the preferred movement direction for each patient and hemisphere. The $\gamma$ ERS was consistently larger for the preferred direction at all three contact pairs in all patients (except one hemisphere in one patient that did not show a significant $\gamma$ synchronization with the supination movement), suggesting that $\gamma$ synchronization representing population activity from different subregions of the pallidum did not vary with movement direction. Instead, we found a significant correlation between the difference in $\gamma$ ERS between pronation and supination movement and the difference in the corresponding movement velocity ( $r=0.711, p=0.01$, data not shown).

\section{Movement onset, velocity, or amplitude}

Behavioral data from our patients revealed a significant increase in movement velocity with increasing amplitude of the movement. To further disentangle the influence of movement velocity and amplitude on $\gamma$ band ERS, we compared the event-related changes in mean $\gamma$ band activity averaged over the three movement conditions aligned to three different time-points (set as time point 0 ): maximum amplitude ( $\max A)$, maximum velocity ( $\operatorname{maxV}$ ), and MO (Fig. 7). Interestingly, the maximum of $\gamma$ band ERS coincided with maxV, suggesting the strongest relationship was between $\gamma$ power and movement speed. Consistently, the largest $\gamma$ ERS occurred when $\gamma$ power was aligned to maxV (Fig. $7)$. Repeated-measures ANOVA revealed a significant effect for TIME POINT $\left(F_{(1.3)}=27.7 ; p<0.001\right.$ Huyhn-Feldt corrected $)$. The mean $\gamma$ band power increase was $40.03 \pm 6.7 \%$ for maxA, $73.9 \pm 11.2 \%$ for $\operatorname{maxV}$, and $54.1 \pm 8.5 \%$ for $\mathrm{MO}$ (each averaged from $-200 \mathrm{~ms}$ to $200 \mathrm{~ms}$ around the respective time-point 0 ). Post hoc Student's $t$ test confirmed significant differences between $\gamma$ ERS during maxA, maxV, and MO $(p<0.05$, Bonferroni corrected).

Next, we correlated the relative change in $\gamma$ band synchronization up to $300 \mathrm{~ms}$ after $\mathrm{MO}$ and $400 \mathrm{~ms}$ around $\operatorname{maxV}$ with the relative difference in movement speed between all three conditions 
A

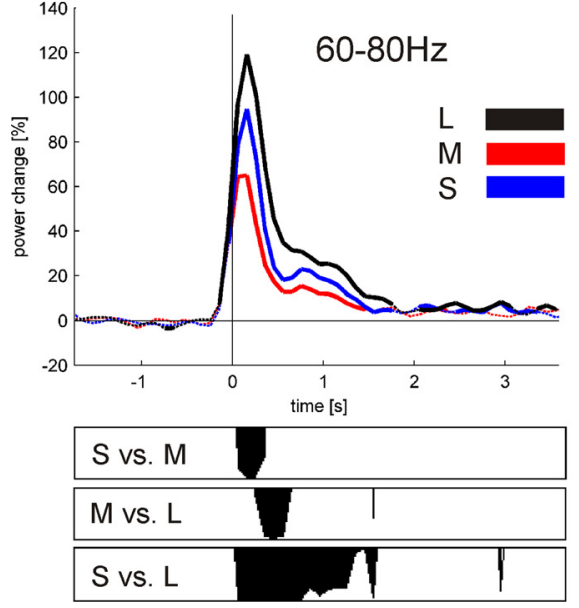

B

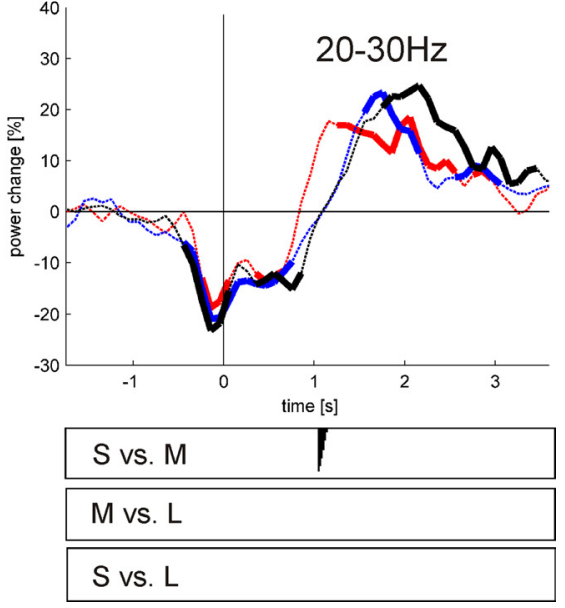

Figure 4. $\quad \boldsymbol{A}, \boldsymbol{B}$, Mean power changes over time for selected contact pairs $(n=36)$ in the $\gamma(\boldsymbol{A} ; 60-80 \mathrm{~Hz})$ and $\beta(\boldsymbol{B} ; 20-30 \mathrm{~Hz})$ band expressed as percentage power change compared with baseline -2 to $-1 \mathrm{~s}$ before $\mathrm{M} 0$ at time point 0 . Note the stepwise increase in $\gamma$ band activity with larger movement amplitudes (small amplitude — blue line, medium amplitude — red line, large amplitude — black line). Thick lines indicate significant changes ( $p<0.05$, FDR corrected) compared with baseline activity. Lower bars indicate significant differences in Wilcoxon's sign-rank test between movement conditions ( $p<0.05$, FDR corrected) in black.

A

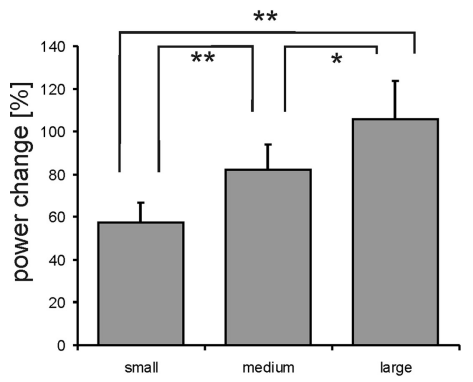

B

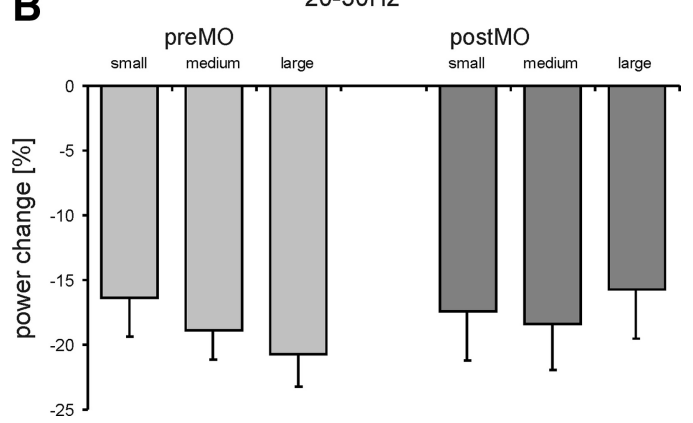

Figure 5. $\quad \boldsymbol{A}$, Mean $\gamma(60-80 \mathrm{~Hz})$ power averaged $0-300 \mathrm{~ms}$ after $\mathrm{M} 0$ for small, medium, and large amplitude movements. $\boldsymbol{B}$, Mean $\beta$ power ( $20-30 \mathrm{~Hz}$ ) power averaged from $-300 \mathrm{~ms}$ to $\mathrm{MO}$ (preM0) and from M0 to $300 \mathrm{~ms}$ after M0 (postM0) for small, medium, and large amplitude movements. ( $n=36$ electrodes, ${ }^{*} p<0.05,{ }^{* *} p<0.001$ Student's $t$ test, Bonferroni corrected).

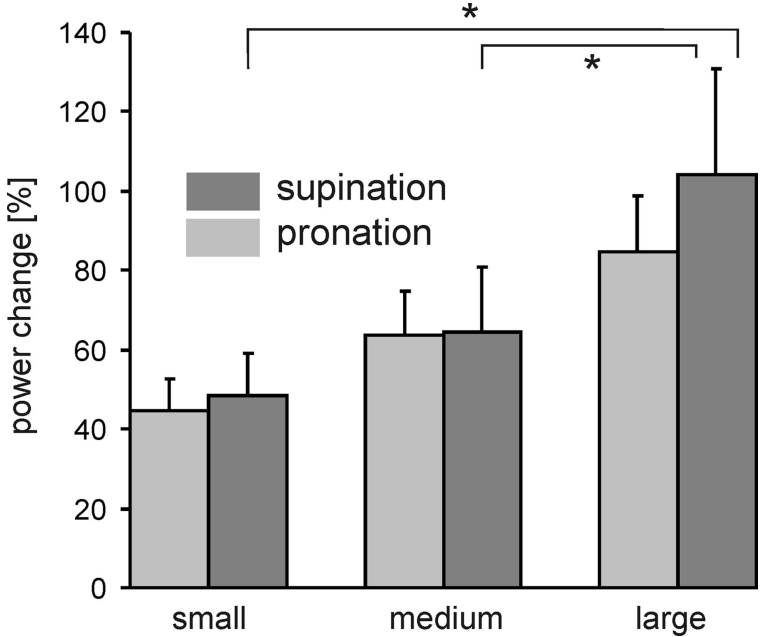

Figure 6. Mean $\gamma(60-80 \mathrm{~Hz})$ power averaged $0-300 \mathrm{~ms}$ after $\mathrm{M} 0$ for small, medium, and large amplitude movements during pronation and supination movements. ${ }^{*} p<0.05$.

(L-M; L-S; M-S). This revealed a significant positive correlation for both $\gamma$ ERS at MO $(r=0.34, \mathrm{p}<0.001)$ and $\operatorname{maxV}(r=0.351, \mathrm{p}<$ 0.001 ) with relative movement speed (Spearman's correlation). To further elucidate this relationship, one subject (case 2) was asked to additionally perform externally paced movements with freely chosen angular degree between $1^{\circ}$ and $90^{\circ}$ with the left forearm leading thus to a higher variability in movement amplitude and velocity on a single trial level. Here, we correlated the $\gamma$ ERS with movement amplitude and velocity on a single trial level. The $60-80 \mathrm{~Hz}$ power in the GPi contralateral to the moved side was averaged $>300 \mathrm{~ms}$ around maxV and maxA of each trial and correlated with the maximum amplitude and velocity of the corresponding movement, respectively. The highest correlation was seen between the $\gamma$ response averaged around maxV and movement velocity $(r=0.45, \mathrm{p}<0.001$, Pearson's correlation, Fig. 8). The correlation between movement amplitude and $\gamma$ ERS averaged around maxA $(r=0.25, p=0.06)$ only showed a trend in this direction. Overall, the amplitude of the movements showed a strong correlation with the movement speed $(r=0.9, \mathrm{p}<0.001$, Pearson's correlation).

\section{Role of proprioceptive afferent feedback on pallidal $\boldsymbol{\gamma}$ ERS}

Overall, during passive movements a small EMG activation was still present, which was significantly smaller compared with the EMG activity during active movements (Fig. 9B). There was no significant difference in mean movement velocity between active and passive movements (paired Student's $t$ test, $\mathrm{p}=0.87$ ). Mean increase in $\gamma$ band activity averaged across the three movement conditions was significantly stronger during active movement trials compared with passive movements (Fig. 9, mean $\gamma$ ERS active: $47.8 \pm 13.3 \%$; passive: $12.2 \pm 6.7 \%, \mathrm{p}=0.007$, two-sided Student's $t$ test), suggesting that afferent feedback had limited influence on the pallidal $\gamma$ ERS.

\section{Discussion}

We have shown that $\gamma$ activity in the GPi LFP contralateral to the moved hand is correlated with movement amplitude and velocity of the hand movement. A stepwise increase in $\gamma$ band activity was seen with larger and faster hand movements. At lower frequencies $(<30$ $\mathrm{Hz}$ ), we observed a symmetrical bilateral decrease that occurred before movement onset and was not modulated by different motor conditions. Thus, the parametric modulation of GPi LFP activity with movement parameters was frequency selective for $\gamma$ activity and occurred around movement onset, supporting a link between $\gamma$ oscillations and kinematics of ongoing movements in patients with dystonia with normal hand motor function. The implication is that the GPi as a major output station of the basal ganglia influences, as indexed by the degree of local $\gamma$ synchronization, the scaling of ongoing movements, whereas a decrease in $\beta$ band activity might be a more general phenomenon during motor preparation that is necessary to allow movement parameterization through other frequencies (such as $\gamma$ band activity) to occur (Brown, 2007).

The $\gamma$ synchronization contralateral to the moved hand is in line with primate studies that have consistently shown 


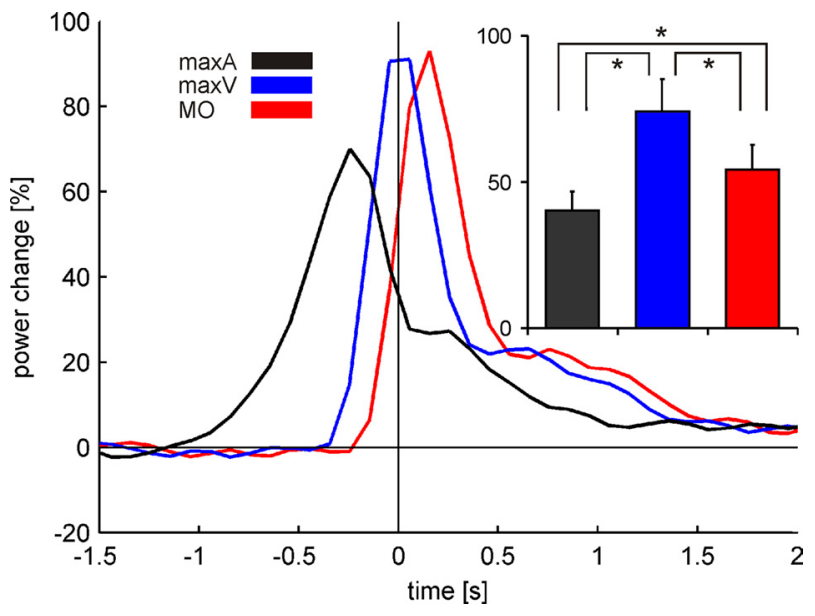

Figure 7. Averaged $60-80 \mathrm{~Hz}$ power over the three movement conditions $(S, M$, and $L)$. The LFP epochs were aligned to maxA (black), $\operatorname{maxV}$ (blue), and MO (red). The peak of the $\gamma$ band aligned to the time point of maximum speed at time point 0 hints at strongest relationship between $\gamma$ power and movement speed. Inset shows mean $\gamma$ power averaged from $-200 \mathrm{~ms}$ to 200 ms around time point $0 .{ }^{*} p<0.05$.

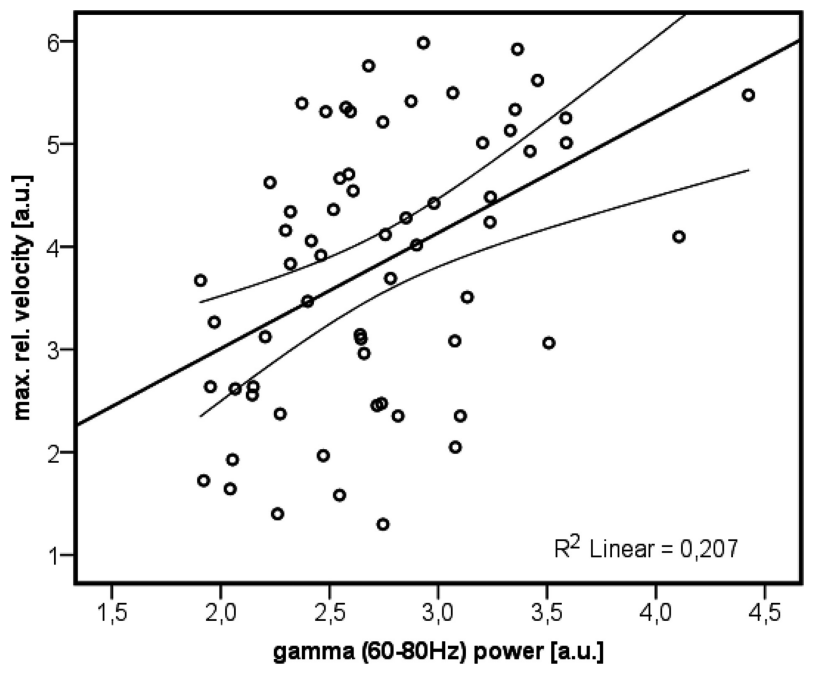

Figure 8. Correlation of maximum relative velocity and mean $60-80 \mathrm{~Hz}$ power $300 \mathrm{~ms}$ around time point max $V$ on single-trial level from right $G$ pi in patient 2. Patient performed left arm movements paced by a cue with freely chosen amplitude and velocity.

movement-related changes of neuronal discharge rate in the GPi during voluntary movements of the contralateral arm (DeLong, 1971; Georgopoulos et al., 1983; Mink and Thach, 1991a,b). Several studies have found that neuronal discharge rate scales with movement direction, amplitude, and/or speed (Georgopoulos et al., 1983; Turner and Anderson, 1997), but others could not attribute discharge rates to movement kinematics (Mink and Thach, 1991b; Brotchie et al., 1991a; Mushiake and Strick, 1995). Local inhibition with muscimol or kainic acid injection into the primate GPi leads to slowness of movements with prolonged movement time, whereas reaction times do not change (Horak and Anderson, 1984; Mink and Thach, 1991c; Desmurget and Turner, 2008). Perimovement increases in pallidal neuronal firing have been mainly observed at movement onset, i.e., rather late compared with activation of motor cortical areas (Georgopoulos et al., 1983; Mitchell et al., 1987; Jaeger et al., 1995; Turner and Anderson, 1997), suggesting its role in modulation of ongoing tasks and scaling of movements without affect-
A
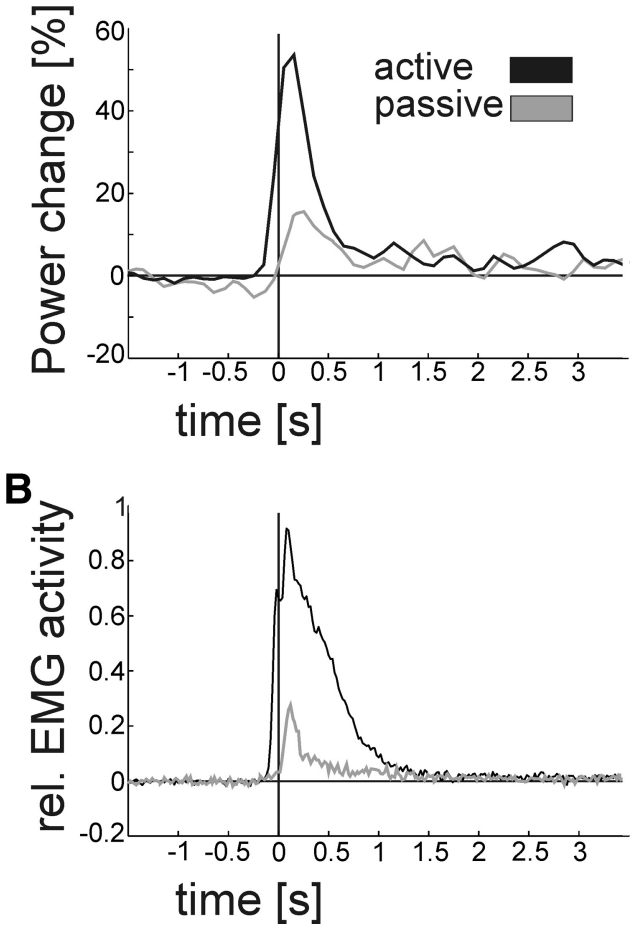

Figure 9. A, Averaged $60-80 \mathrm{~Hz}$ power during active (black) and passive (gray) movements. Data are from 4 patients and $6 \mathrm{GPi}(n=6)$. B, Averaged relative rectified EMG activity during active and passive movements. Data are averaged across the three movement conditions.

ing the initiation and sequential organization of programmed motor output. Moreover, a correlation between BG activity and the velocity or amplitude of the movement was shown in normal subjects using PET (Thobois et al., 2007; Turner et al., 2003) or fMR imaging (Grafton and Tunik, 2011). Prodoehl et al. (2009) proposed that dorsal BG (including the internal pallidum) also regulate the parametrization of grip force, whereas changes in fMRI BOLD signal in the ventral BG scale with the prediction of force amplitude (Vaillancourt et al., 2004, 2007). Based on these observations it has been suggested that basal ganglia output regulates (among other behavioral and sensory dimensions) movement gain (Turner and Desmurget, 2010). In this framework, we would tentatively interpret pallidal $\gamma$ synchronization that occurred around movement onset in our patients to index BG influence on movement gain. However, it has to be pointed out that other models of BG function have been put forward, suggesting a major contribution of the BG to movement selection and initiation (Mink, 2003; Houk and Wise, 1995; Houk et al., 2007), encoding of context-dependent information of movements, cognitive aspects of motor control (Brotchie et al., 1991b; Mushiake and Strick, 1995; Pasquereau et al., 2007), but also motor learning (Doyon et al., 2009) and feedback control of movements (Brainard, 2004; Brown et al., 2006). Our results showing a parametric modulation of pallidal $\gamma$ synchronization with increasing velocity of the movement provide support for the scaling hypothesis and related cost functions, and raise the possibility that $\gamma$ activity may be involved in mediating this scaling. Gamma activity may facilitate information coding and processing (Pogosyan et al., 2006) as well as "motor binding" (Engel et al., 2005), i.e., supporting binding of distributed responses and selection of specific motor parameters. The above does not necessarily imply that $\gamma$ activity represents explicit motor processing, and $\gamma$ synchrony may serve 
different functions in the visual and motor system. Moreover, increased $\gamma$ activity has been described in REM sleep without limb movement and following startling stimuli. Both are more likely related to arousal or attentional changes that influence gain leading to greater energization of the movement (Kempf et al., 2009).

A general mechanism by which the basal ganglia may regulate motor control was recently suggested as being the scaling of the effort or motor energy invested in a movement (Mazzoni et al., 2007). In this model of motor control, the effort variable (motor cost) is dependent on the velocity and/or amplitude of movements. Our results would also be in line with the assumption that basal ganglia control motor costs rather than motor parameters, since other kinematic parameters such as movement direction were controlled independently (Vindras et al., 2005) and did not influence the degree of $\gamma$ synchronization in our patients. However, the present study was not intended to disentangle correlates of motor energy and movement velocity, and future studies should evaluate the potential role of pallidal $\gamma$ activity for modulation of motor performance according to energetic or other costs to the subject.

In this regard it is interesting to note that motor slowing and reduced amplitude of movements has been observed in patients treated with bilateral high-frequency stimulation of the GPi for Huntington's disease (Moro et al., 2004), dystonia (Ostrem et al., 2007), or Tourette's syndrome (Diederich et al., 2005), possibly induced by interference of high-frequency DBS with physiological pallidal motor output.

There is increasing evidence of movement-related $\gamma$ band synchronization as a feature of basal ganglia activity that is common across different movement disorders. Gamma band ERS has been recorded from GPi in both generalized and focal dystonia (Brücke et al., 2008; Liu et al., 2008), from the subthalamic nucleus in treated and untreated PD patients, and in patients with nonparkinsonian tremor (Androulidakis et al., 2007; Kempf et al., 2007). The movement-related $\gamma$ increase was strictly lateralized in patients with dystonia, and lateralization was facilitated by dopaminergic treatment in $\mathrm{PD}$, suggesting a more physiological pattern of motor processing promoted by therapeutic intervention in PD (Androulidakis et al., 2007). Here, our data provide further support to the idea that movement-related lateralized $\gamma$ band synchronization is a feature of physiological basal ganglia activity encoding information about movement kinematics or energization of movement.

These observations from deep brain recordings are paralleled by activity at the cortical level obtained in ECoG (electrocorticography) in patients with epilepsy (Crone et al., 1998; Crone et al., 2006; Mehring et al., 2004) and in normal subjects using MEG and EEG (Cheyne et al., 2008; Waldert et al., 2008; Ball et al., 2008; Huo et al., 2010) that have demonstrated the occurrence of $\gamma$ oscillations in a similar frequency range $(60-150 \mathrm{~Hz})$ in the primary motor cortex during movement onset contralateral to the moved limb. More recently, Muthukumaraswamy (2010) has revealed that motor cortical $\gamma$ synchronization is greater with larger movements with a time to peak at $\sim 137 \mathrm{~ms}$ after EMG onset, not sustained during isometric contraction, and absent during passive movements. These results are strikingly similar to our observations from deep brain recordings, strongly indicating that $\gamma$ synchronization at cortical and basal ganglia levels reflects active motor processing at a relatively late stage of motor control. Additionally, the significant correlation of movement velocity and $\gamma$ synchronization in our data suggests that $\gamma$ oscillations may encode specific kinematic parameters such as movement velocity or, more general phenomena, such as motor gain and energization of movement. However, whether cortical motor areas drive basal ganglia output at $\gamma$ frequency or whether movement-related $\gamma$ synchronization has been generated locally in the GPi cannot be answered since EEG was not available in our patients due to surgical dressings.

We have further shown that pallidal $\gamma$ synchronization is related to the motor output and is not a result of proprioceptive feedback, since $\gamma$ increase in pallidal LFP activity during passive movements was significantly smaller compared with active movement. This is in line with results of a previous study using a block design of different sensorimotor conditions that revealed $\gamma$ synchronization with voluntary but not passive movements in patients with dystonia (Liu et al., 2008).

An important question remains as to how the underlying disease may have influenced our results. We have to bear in mind that results are obtained in patients with movement disorders, thus there is no certainty that findings are physiological. However, to avoid major confounds induced by abnormal hand movements [shown to induce $\gamma$ activity in generalized dystonia patients, see the study by Liu et al. (2008)], we have only included patients with normal hand motor function in our study. It also has to be noted that abnormal muscle activity has been related to increased low-frequency activity $(4-10 \mathrm{~Hz})$ in dystonia patients, and $\gamma$ band activity is usually not found in the resting state pallidal LFP activity (Liu et al., 2008; Sharott et al., 2008).

In summary, our study provides evidence that basal ganglia output encodes information related to motor gain and that this is at least partly indexed by the degree of pallidal $\gamma$ band synchronization. This raises the possibility that $\gamma$ activity may play a role in the communication between neuronal populations during movement execution and regulation of movement gain.

\section{References}

Alegre M, Alonso-Frech F, Rodríguez-Oroz MC, Guridi J, Zamarbide I, Valencia M, Manrique M, Obeso JA, Artieda J (2005) Movement-related changes in oscillatory activity in the human subthalamic nucleus: ipsilateral vs. contralateral movements. Eur J Neurosci 22:2315-2324.

Androulidakis AG, Kühn AA, Chen CC, Blomstedt P, Kempf F, Kupsch A, Schneider GH, Doyle L, Dowsey-Limousin P, Hariz MI, Brown P (2007) Dopaminergic therapy promotes lateralized motor activity in the subthalamic area in Parkinson's disease. Brain 130:457-468.

Ball T, Demandt E, Mutschler I, Neitzel E, Mehring C, Vogt K, Aertsen A, Schulze-Bonhage A (2008) Movement related activity in the high gamma range of the human EEG. Neuroimage 41:302-310.

Bauer M, Oostenveld R, Peeters M, Fries P (2006) Tactile spatial attention enhances gamma-band activity in somatosensory cortex and reduces lowfrequency activity in parieto-occipital areas. J Neurosci 26:490-501.

Benjamini Y, Hochberg Y (1995) Controlling the false discovery rate: a practical and powerful approach to multiple testing. J R Stat Soc B Methodol 57:289-300.

Berman BD, Starr PA, Marks WJ Jr, Ostrem JL (2009) Induction of bradykinesia with pallidal deep brain stimulation in patients with cranialcervical dystonia. Stereotact Funct Neurosurg 87:37-44.

Blahak C, Capelle HH, Baezner H, Kinfe TM, Hennerici MG, Krauss JK (2011) Micrographia induced by pallidal DBS for segmental dystonia: a subtle sign of hypokinesia? J Neural Transm 118:549-553.

Brainard MS (2004) Contributions of the anterior forebrain pathway to vocal plasticity. Ann N Y Acad Sci 1016:377-394.

Brotchie P, Iansek R, Horne MK (1991a) Motor function of the monkey globus pallidus. 1. Neuronal discharge and parameters of movement. Brain 114:1667-1683.

Brotchie P, Iansek R, Horne MK (1991b) Motor function of the monkey globus pallidus. 2. Cognitive aspects of movement and phasic neuronal activity. Brain 114:1685-1702.

Brown P (2003) Oscillatory nature of human basal ganglia activity: relationship to the pathophysiology of Parkinson's disease. Mov Disord 18:357-363. 
Brown P (2007) Abnormal oscillatory synchronisation in the motor system leads to impaired movement. Curr Opin Neurobiol 17:656-664.

Brown P, Oliviero A, Mazzone P, Insola A, Tonali P, Di Lazzaro V (2001) Dopamine dependency of oscillations between subthalamic nucleus and pallidum in Parkinson's disease. J Neurosci 21:1033-1038.

Brown P, Chen CC, Wang S, Kühn AA, Doyle L, Yarrow K, Nuttin B, Stein J, Aziz T (2006) Involvement of human basal ganglia in offline feedback control of voluntary movement. Curr Biol 16:2129-2134.

Brücke C, Hübl J, Kempf F, Krauss JK, Yarrow K, Kupsch A, Schneider G-H, Brown P, Kühn AA (2008a) Pallidal gamma activity is correlated to movement amplitude in patients with dystonia. Clin Neurophysiol 119 [S1]:49.

Brücke C, Kempf F, Kupsch A, Schneider GH, Krauss JK, Aziz T, Yarrow K, Pogosyan A, Brown P, Kühn AA (2008b) Movement-related synchronization of gamma activity is lateralized in patients with dystonia. Eur J Neurosci 27:2322-2329.

Buzsáki G (2006) Rhythms of the brain. Oxford: Oxford UP.

Cassidy M, Mazzone P, Oliviero A, Insola A, Tonali P, Di Lazzaro V, Brown P (2002) Movement-related changes in synchronization in the human basal ganglia. Brain 125:1235-1246.

Cheyne D, Bells S, Ferrari P, Gaetz W, Bostan AC (2008) Self-paced movements induce high-frequency gamma oscillations in primary motor cortex. Neuroimage 42:332-342.

Crone NE, Miglioretti DL, Gordon B, Lesser RP (1998) Functional mapping of human sensorimotor cortex with electrocorticographic spectral analysis. II. Event-related synchronization in the gamma band. Brain 121:2301-2315.

Crone NE, Sinai A, Korzeniewska A (2006) High-frequency gamma oscillations and human brain mapping with electrocorticography. Prog Brain Res 159:275-295.

DeLong MR (1971) Activity of pallidal neurons during movement. J Neurophysiol 34:414-427.

Desmurget M, Turner RS (2008) Testing basal ganglia motor functions through reversible inactivations in the posterior internal globus pallidus. J Neurophysiol 99:1057-1076.

Diederich NJ, Kalteis K, Stamenkovic M, Pieri V, Alesch F (2005) Efficient internal pallidal stimulation in Gilles de la Tourette syndrome: a case report. Mov Disord 20:1496-1499.

Doyon J, Bellec P, Amsel R, Penhune V, Monchi O, Carrier J, Lehéricy S, Benali H (2009) Contributions of the basal ganglia and functionally related brain structures to motor learning. Behav Brain Res 199:61-75.

Engel AK, Fries P, Singer W (2001) Dynamic predictions: oscillations and synchrony in top-down processing. Nat Rev Neurosci 2:704-716.

Engel AK, Moll CK, Fried I, Ojemann GA (2005) Invasive recordings from the human brain: clinical insights and beyond. Nat Rev Neurosci 6:35-47.

Fogelson N, Pogosyan A, Kühn AA, Kupsch A, van Bruggen G, Speelman H, Tijssen M, Quartarone A, Insola A, Mazzone P, Di Lazzaro V, Limousin P, Brown P (2005) Reciprocal interactions between oscillatory activities of different frequencies in the subthalamic region of patients with Parkinson's disease. Eur J Neurosci 22:257-266.

Georgopoulos AP, DeLong MR, Crutcher MD (1983) Relations between parameters of step-tracking movements and single cell discharge in the globus pallidus and subthalamic nucleus of the behaving monkey. J Neurosci 3:1586-1598.

Grafton ST, Tunik E (2011) Human basal ganglia and the dynamic control of force during on-line corrections. J Neurosci 31:1600-1605.

Horak FB, Anderson ME (1984) Influence of globus pallidus on arm movements in monkeys. I. Effects of kainic acid-induced lesions. J Neurophysiol 52:290-304.

Houk JC, Wise SP (1995) Distributed modular architectures linking basal ganglia, cerebellum, and cerebral cortex: their role in planning and controlling action. Cereb Cortex 5:95-110.

Houk JC, Bastianen C, Fansler D, Fishbach A, Fraser D, Reber PJ, Roy SA, Simo LS (2007) Action selection and refinement in subcortical loops through basal ganglia and cerebellum. Philos Trans R Soc Lond B Biol Sci 362:1573-1583.

Huo X, Xiang J, Wang Y, Kirtman EG, Kotecha R, Fujiwara H, Hemasilpin N, Rose DF, Degrauw T (2010) Gamma oscillations in the primary motor cortex studied with MEG. Brain Dev 32:619-624.

Jaeger D, Gilman S, Aldridge JW (1995) Neuronal activity in the striatum and pallidum of primates related to the execution of externally cued reaching movements. Brain Res 694:111-127.
Jensen O, Kaiser J, Lachaux JP (2007) Human gamma-frequency oscillations associated with attention and memory. Trends Neurosci 30:317-324.

Kempf F, Kühn AA, Kupsch A, Brücke C, Weise L, Schneider GH, Brown P (2007) Premovement activities in the subthalamic area of patients with Parkinson's disease and their dependence on task. Eur J Neurosci 25:3137-3145.

Kempf F, Brücke C, Salih F, Trottenberg T, Kupsch A, Schneider GH, Doyle Gaynor LM, Hoffmann KT, Vesper J, Wöhrle J, Altenmüller DM, Krauss JK, Mazzone P, Di Lazzaro V, Yelnik J, Kühn AA, Brown P (2009) Gamma activity and reactivity in human thalamic local field potentials. Eur J Neurosci 29:943-953.

Kühn AA, Williams D, Kupsch A, Limousin P, Hariz M, Schneider GH, Yarrow K, Brown P (2004) Event-related beta desynchronization in human subthalamic nucleus correlates with motor performance. Brain 127:735-746.

Kupsch A, Benecke R, Müller J, Trottenberg T, Schneider GH, Poewe W, Eisner W, Wolters A, Müller JU, Deuschl G, Pinsker MO, Skogseid IM, Roeste GK, Vollmer-Haase J, Brentrup A, Krause M, Tronnier V, Schnitzler A, Voges J, Nikkhah G, et al. (2006) Pallidal deep-brain stimulation in primary generalized or segmental dystonia. $\mathrm{N}$ Engl J Med 355:1978-1990

Lalo E, Thobois S, Sharott A, Polo G, Mertens P, Pogosyan A, Brown P (2008) Patterns of bidirectional communication between cortex and basal ganglia during movement in patients with Parkinson disease. J Neurosci 28:3008-3016.

Liu X, Wang S, Yianni J, Nandi D, Bain PG, Gregory R, Stein JF, Aziz TZ (2008) The sensory and motor representation of synchronized oscillations in the globus pallidus in patients with primary dystonia. Brain 131:1562-1573.

Mazzoni P, Hristova A, Krakauer JW (2007) Why don't we move faster? Parkinson's disease, movement vigor, and implicit motivation. J Neurosci 27:7105-7116.

Mehring C, Nawrot MP, de Oliveira SC, Vaadia E, Schulze-Bonhage A, Aertsen A, Ball T (2004) Comparing information about arm movement direction in single channels of local and epicortical field potentials from monkey and human motor cortex. J Physiol Paris 98:498-506.

Mink JW (2003) The Basal Ganglia and involuntary movements: impaired inhibition of competing motor patterns. Arch Neurol 60:1365-1368.

Mink JW, Thach WT (1991a) Basal ganglia motor control. I. Nonexclusive relation of pallidal discharge to five movement modes. J Neurophysiol 65:273-300.

Mink JW, Thach WT (1991b) Basal ganglia motor control. II. Late pallidal timing relative to movement onset and inconsistent pallidal coding of movement parameters. J Neurophysiol 65:301-329.

Mink JW, Thach WT (1991c) Basal ganglia motor control. III. Pallidal ablation: normal reaction time, muscle cocontraction, and slow movement. J Neurophysiol 65:330-351.

Mitchell SJ, Richardson RT, Baker FH, DeLong MR (1987) The primate globus pallidus: neuronal activity related to direction of movement. Exp Brain Res 68:491-505.

Moro E, Lang AE, Strafella AP, Poon YY, Arango PM, Dagher A, Hutchison WD, Lozano AM (2004) Bilateral globus pallidus stimulation for Huntington's disease. Ann Neurol 56:290-294.

Mushiake H, Strick PL (1995) Pallidal neuron activity during sequential arm movements. J Neurophysiol 74:2754-2758.

Muthukumaraswamy SD (2010) Functional properties of human primary motor cortex gamma oscillations. J Neurophysiol 104:2873-2885.

Ostrem JL, Marks WJ Jr, Volz MM, Heath SL, Starr PA (2007) Pallidal deep brain stimulation in patients with cranial-cervical dystonia (Meige syndrome). Mov Disord 22:1885-1891.

Pasquereau B, Nadjar A, Arkadir D, Bezard E, Goillandeau M, Bioulac B, Gross CE, Boraud T (2007) Shaping of motor responses by incentive values through the basal ganglia. J Neurosci 27:1176-1183.

Pfurtscheller G, Graimann B, Huggins JE, Levine SP, Schuh LA (2003) Spatiotemporal patterns of beta desynchronization and gamma synchronization in corticographic data during self-paced movement. Clin Neurophysiol 114:1226-1236.

Pogosyan A, Kühn AA, Trottenberg T, Schneider GH, Kupsch A, Brown P (2006) Elevations in local gamma activity are accompanied by changes in the firing rate and information coding capacity of neurons in the region of the subthalamic nucleus in Parkinson's disease. Exp Neurol 202:271-279. 
Prodoehl J, Corcos DM, Vaillancourt DE (2009) Basal ganglia mechanisms underlying precision grip force control. Neurosci Biobehav Rev 33:900-908.

Reese R, Gruber D, Schoenecker T, Bäzner H, Blahak C, Capelle HH, Falk D, Herzog J, Pinsker MO, Schneider GH, Schrader C, Deuschl G, Mehdorn HM, Kupsch A, Volkmann J, Krauss JK (2011) Long-term clinical outcome in meige syndrome treated with internal pallidum deep brain stimulation. Mov Disord 26:691-698.

Schoffelen JM, Oostenveld R, Fries P (2005) Neuronal coherence as a mechanism of effective corticospinal interaction. Science 308:111-113.

Schönecker T, Kupsch A, Kühn AA, Schneider GH, Hoffmann KT (2009) Automated optimization of subcortical cerebral MR imaging-atlas coregistration for improved postoperative electrode localization in deep brain stimulation. AJNR Am J Neuroradiol 30:1914-1921.

Sharott A, Grosse P, Kühn AA, Salih F, Engel AK, Kupsch A, Schneider GH, Krauss JK, Brown P (2008) Is the synchronization between pallidal and muscle activity in primary dystonia due to peripheral afferance or a motor drive? Brain 131:473-484.

Silberstein P, Kühn AA, Kupsch A, Trottenberg T, Krauss JK, Wöhrle JC, Mazzone P, Insola A, Di Lazzaro V, Oliviero A, Aziz T, Brown P (2003) Patterning of globus pallidus local field potentials differs between Parkinson's disease and dystonia. Brain 126:2597-2608.

Thobois S, Ballanger B, Baraduc P, Le Bars D, Lavenne F, Broussolle E, Desmurget M (2007) Functional anatomy of motor urgency. Neuroimage $37: 243-252$.
Turner RS, Anderson ME (1997) Pallidal discharge related to the kinematics of reaching movements in two dimensions. J Neurophysiol 77:10511074 .

Turner RS, Desmurget M (2010) Basal ganglia contributions to motor control: a vigorous tutor. Curr Opin Neurobiol 20:704-716.

Turner RS, Desmurget M, Grethe J, Crutcher MD, Grafton ST (2003) Motor subcircuits mediating the control of movement extent and speed. J Neurophysiol 90:3958-3966.

Vaillancourt DE, Mayka MA, Thulborn KR, Corcos DM (2004) Subthalamic nucleus and internal globus pallidus scale with the rate of change of force production in humans. Neuroimage 23:175-186.

Vaillancourt DE, Yu H, Mayka MA, Corcos DM (2007) Role of the basal ganglia and frontal cortex in selecting and producing internally guided force pulses. Neuroimage 36:793-803.

Varela F, Lachaux JP, Rodriguez E, Martinerie J (2001) The brainweb: phase synchronization and large-scale integration. Nat Rev Neurosci 2:229_ 239.

Vindras P, Desmurget M, Viviani P (2005) Error parsing in visuomotor pointing reveals independent processing of amplitude and direction. J Neurophysiol 94:1212-1224.

Waldert S, Preissl H, Demandt E, Braun C, Birbaumer N, Aertsen A, Mehring C (2008) Hand movement direction decoded from MEG and EEG. J Neurosci 28:1000-1008. 